\title{
La Sociología del crimen y la violencia en América Latina \\ Un campo fragmentado
}

Arturo Alvarado*

https://orcid.org/0000-0002-6922-3507

Introducción

El presente artículo es una genealogía de los estudios sobre Sociología del crimen y la violencia en América Latina. El origen de este campo marcha de forma paralela al desarrollo e institucionalización de la disciplina en la región, cuando surge una comunidad epistémica en estos temas y un campo autónomo de otras disciplinas. Para este trabajo utilizamos la categoría sociológica de crimen como un sinónimo tanto de la delincuencia como de la violencia, y consiste en una desviación y una transgresión a comportamientos sociales y a reglas establecidas en convenciones sociales, en códigos criminales, nacionales o internacionales, no importa cuáles sean los valores prevalentes en nuestras heterogéneas sociedades, pues la constitución del crimen es un proceso social, en el cual se construyen tanto el delito como el delincuente. La categoría incluye un comportamiento violento en el sentido y propósito de violar reglas legales y sociales y producir (o intentar) un daño al bienestar personal o colectivo. El presente artículo no cubre la literatura general sobre criminalidad que no es parte central de la Sociología del Crimen, como la que proviene de otras áreas sociológicas, como la Jurídica, la Política, la Criminología, la Salud Pública o la Epidemiología (particularmente la definición de violencia y los estudios de homicidios) ${ }^{2}$.

* El Colegio de México, Ciudad de México, México.

1. Agradecimento especial ao Rafael Montovani, doutor em sociologia pela UsP, pela revisão do texto.

2. La Sociología y la Criminología en América Latina han corrido por senderos entrelazados que en algu- 
El desarrollo de este campo podría parecer una tendencia continental, no obstante, en realidad, cada país ha trazado su propia ruta, acorde con los problemas nacionales, aun cuando no sean los temas dominante regionales o estén fragmentados.

La gran mayoría de las investigaciones empíricas fueron producidas en instituciones públicas y en esos entornos surgieron las comunidades de científicos sociales, en sus plataformas académicas, en revistas, publicaciones de libros y en los proyectos financiados por sus gobiernos, con algunas excepciones que fueron impulsadas por fundaciones internacionales, como la Organización de las Naciones Unidas para la Educación, la Ciencia y la Cultura (Unesco), el Consejo Latinoamericano de Ciencias Sociales (Clacso), la Facultad Latinoamericana de Ciencias Sociales (Flacso) o la Fundación Ford.

En el presente artículo presentamos cuatro rutas y tres momentos del desarrollo histórico de la Sociología del Crimen en América Latina. Para identificar el campo y su magnitud, comenzamos por diseñar un esquema analítico en donde agrupamos los principales temas que han ocupado a la literatura de lo criminal o lo violento o lo delictual, así como del delincuente, el desviado o el criminal, sus motivos y sus formas de organización y, finalmente, las victimas. Para el presente trabajo comparamos otros artículos que analizan los campos temáticos de la Sociología del Crimen (véase: Adorno, 1993, Zaluar, 1999; Barreira y Adorno, 2010; Gabaldón, 2010; Lima y Ratton, 2011; Freitas y Ribeiro, 2013; Imbusch et al., 2011; Campos y Alvarez, 2017; Santos y Barreira, 2016; Briceño, 2016; Ratton, 2018; Misse, 2019; Fellini Fachinetto et al., 2020), que agrupan la producción intelectual y académica en las seis décadas pasadas ${ }^{3}$. A partir de esto, efectuamos una serie de búsquedas de las

nos casos se juntan, en otros se bifurcan, han tejido investigaciones sobre muchos temas comunes y, sin embargo, cada una ha creado sus propios campos y sus comunidades no son las mismas, incluso sus explicaciones del delito compiten entre sí. Ambas surgen de orientación jurídicas, mas la Criminología aprovechó la expansión del derecho liberal en el siglo xx, así como los giros en la criminología crítica hacia los años 1980s, que impulsaron los estudios de las penas, la victimología, las instituciones penales y las políticas de prevención con vertientes originales en el subcontinente. Desde entonces se ha alimentado de un corpus de literatura distinto (más cercano a la Sociología Jurídica) y mantiene preocupaciones en problemas donde la Sociología no ha ahondado, aunque muchos autores la combinen o la confundan. Los postulados teóricos sobre los cuales explican el delito (sus causas y el sistema de penas, consideran el delito asociado con el delincuente) son jurídicos y criminógenos, no sociológicos. Sus intereses se concentran en el sistema penal, en los estados penales (considerado desigual, injusto, un sistema de represión de las clases dominantes), y en críticas de las acciones y políticas penales del estado. Entre los autores que han tenido una presencia continua en los últimos 40 años están C. Birkbech, E. Carranza, L. Gabaldón, R. Del Olmo, L. De Castro Anyar. También Cohen, E. Zaffaroni, A. Baratta Roberto Bergalli, Juan Bustos Ramirez, M. Beloff, E. García Méndez y otros seguidores de la criminología crítica que citamos en el texto, como J. Pegoraro (Gabaldón, 2010, pp. 256 y ss.).

3. En un artículo reciente Fellini Fascinetto et al. (2020) proponen una definición del campo que abarca la "Sociología de la violencia, el crimen y la justicia criminal" (p. 1), porque incorporan temas del sistema 
publicaciones por internet, en catálogos de bibliotecas, así como la revisión de las revistas latinoamericanas que tienen más circulación de estos tópicos en la región, en idiomas castellano y portugués, principalmente. El resultado de las búsquedas muestra la cantidad, variedad e incremento de las publicaciones. Pero también presenta inconsistencias en la clasificación y el número preciso de artículos divulgados (ver cuadro anexo $)^{4}$. De esta búsqueda se confirmó el conjunto de dimensiones diseñadas para el trabajo, así como un grupo de cuatro países que resultaron con una mayor plataforma institucional para difundir sus investigaciones, con comunidades científicas consolidadas y con financiamiento a su producción. Por ejemplo, en la búsqueda de tres sitios (Redalyc, Scielo y WorldCat), se registraron 5.637 artículos

de justicia, así como de derechos humanos y políticas de seguridad pública. Esto abre una puerta a la interacción entre Sociología Jurídica o del derecho, la Criminología y la Ciencia Política, haciendo mucho más compleja una genealogía de la disciplina. El campo de temas es más amplio que las cuatro grandes dimensiones propuestas por Adorno (1993), las nueve categorías de Zaluar (1999), o Campos y Alvarez (2017) así como Ratton (2018). Además de ofrecer una excelente revisión del debate sobre el campo, proponen también una idea de linajes de la mayoría de los autores considerados pioneros (ver más adelante) de acuerdo con la cantidad de estudiantes dirigidos (orientados) en sus tesis y sugieren tres descendencias (o generaciones), lo que muestra la amplitud disciplinaria de los nuevos investigadores. Su balance sobre la temática en Brasil es basto (op. cit.: Cuadro de páginas 4-5), pero también muestran la escasez de trabajos similares para la región. Solo Imbusch et al. (2011) y Tavares Dos Santos y Barreira (2016) ensayan una exploración para América Latina, con las limitaciones propias de las fuentes y los idiomas.

4. Para calcular una estimación general de las publicaciones, se realizaron varias búsquedas en los principales sitios de revistas y catálogos de la región: Redalyc, Dialnet, Scielo, Doaj, WorlCat, ResearchGate, Google Scholar, Fuente Académica. Posteriormente se examinaron los índices de algunas de las revistas más conocidas en los temas y de mayor trayectoria. No utilizamos las clasificaciones de revistas de distintos sitios y empresas porque no hay consenso sobre estas en América Latina. Las dimensiones buscadas fueron: violencia en general, homicidios, delincuencia, crimen organizado, narcotráfico, violencia urbana, contrabando, piratería, tráfico de armas, violaciones a derechos humanos, desplazamiento forzado, seguridad ciudadana, policía y prisiones. Las categorías fueron agrupadas posteriormente en los temas sociológicos del artículo. En algunos casos se hicieron búsquedas cruzadas o con límites por año, por tema (en español y portugués), por país y región (A.L.). Los resultados son un indicador aproximado de las publicaciones. Los registros que cada fuente anota no son homogéneos y hay varios problemas de especificación de las búsquedas. Por ejemplo, en el filtro "años", las publicaciones varían y la mayoría de los servidores no cuenta con información antes de 1990. La mayoría de los buscadores no permite búsquedas por país y región y muchos más compilan indistintamente revistas con libros. En varias ocasiones encontramos errores o erratas en las clasificaciones, en los nombres de autores y el uso del principal apellido de autor varía entre fuentes y entre países. Algunos buscadores adhieren automáticamente términos proxi en las búsquedas de vocablos simples. Por ejemplo, para la categoría "violencia" incluye numerosos temas asociados, como jóvenes, noviazgo, pareja, familia, etc. Cabe recalcar que no hay vínculos entre los distintos sitios de revistas y que sitios como Google Académico mezclan muchos temas. Por lo tanto, estos datos son considerados como indicativos de la producción, pero no determinantes del número real de publicaciones. Tampoco nos permite identificar un número claro de autores por país, lo cual sería útil para conocer las comunidades académicas de los países. Este será un trabajo futuro importante que permitirá construir redes y comunidades. Agradezco a Denis Salazar, a Melissa Sánchez y Daniel Hernández el apoyo para las búsquedas. 
y libros sobre violencia; 734 sobre homicidios y 704 sobre narcotráfico. Pero en ese mismo momento Dialnet arrojó 23.862 publicaciones sobre violencia. Ningún buscador encontró registros antes de 1990 y la mayoría están concentrados entre los años 2000 y 2020. La producción sociológica en la región tiene presencia en más de 20 países $^{5}$. Entre ellos, cuatro países emergen como los países con mayor contribución al campo; estos son Brasil (763 registros), México (3.956), Argentina (1.063) y Colombia (3.255). En contraste con las cifras de las revistas, un recuento realizado en Brasil por Barreira y Adorno (2010) estimó más de 2 mil artículos publicados en revistas en su país sobre este campo.

Para esta genealogía revisamos con mayor detalle otras publicaciones y el registro de grupos de investigación. Los cuatro países iniciaron trabajos “tempranos” en la subdisciplina. Por ejemplo, a fines de la década de 1990 en Colombia la subdisciplina ocupaba el $8^{\circ}$ lugar entre los estudios financiados por Colciencias (Rodríguez, 1996, en: Sandoval, 2014). Pero la mayoría vio interrumpidos sus avances por eventos políticos cruentos, como las dictaduras, la represión y censura a la ciencia y a la academia entre los años sesenta y ochenta. Los cientistas sociales de este campo han sostenido una relación ambivalente o conflictiva con sus regímenes. La represión en el pasado autoritario forjó un pensamiento independiente y crítico del gobierno que se mantiene junto con su simpatía por la democracia.

Argentina, Colombia, Brasil y México ${ }^{6}$ han consolidado el campo y la comunidad científica a lo largo de tres períodos que abarcan 60 años, y esto se configura con cuatro propiedades: (i) una comunidad epistémica que comparte intereses, algunos supuestos y definiciones de delitos y semánticas, algún conocimiento de teorías sociales sobre el tema, que se refleja en asociaciones con vínculos fuertes como la Sociedad Brasileña de Sociología y que genera las primeras ideas, interpretaciones y estudios empíricos; (ii) una plataforma institucional para la investigación, la enseñanza y la divulgación del campo, particularmente con la fundación de carreras de Sociología, facultades e institutos de investigación, sobre todo con postgrados; (iii) un conjunto de publicaciones y (iv) un conjunto de "hechos criminales" que ha tenido un crecimiento exponencial. Su desarrollo abarca tres etapas. La primera es de formulación del campo problemático, con una primera generación de especialistas; la

5. Una búsqueda con los mismos criterios en diferentes días durante cuatro semanas arrojaba resultados diferentes. Redalyc arrojó en otro momento un resultado para la categoría de "violencia", de 11.147.

6. Venezuela ha tenido un desarrollo importante en las dos primeras etapas de la Sociología, con grupos de investigadores consolidados. También ha creado revistas de ciencias sociales importantes, pero en el período contemporáneo resulta difícil buscar literatura sobre su producción académica. En países de Centroamérica existen varios trabajos, pero no hay una escuela o una comunidad consolidada y varios trabajos importantes son de autores externos, con importantes excepciones. En Guatemala destacan trabajos en etapas “tempranas", por ejemplo, los de Figueroa sobre vigilantismo (1991). 
segunda, la consolidación de una red de expertos, un conjunto de ensayos empíricos con discusiones disciplinarias sólidas, y el tercer período, que llamaré contemporáneo, con una red densa de expertos que cubren la gran mayoría de las dimensiones que seleccionamos en donde cuentan con una infraestructura y estudios empíricos cada vez más rigurosos.

Temas, orientaciones teóricas, independencia y pertinencia de la subdisciplina

La sociología del delito en la región es amplia en temas y enfoques. Todas nuestras sociedades han tenido ciertos niveles de crimen a lo largo del siglo veinte, acompañando procesos de transformación profunda, tales como la transición de una sociedad rural a una predominantemente urbana, la transformación de la economía y el empleo en la sociedad capitalista, la expansión de la educación, junto con la permanencia de distintos tipos de desigualdades, la evolución de la pobreza y la evolución de los estados nacionales, particularmente de sus aparatos coercitivos y de castigo.

El delito es una violación a una regla social y una transgresión a un código legal. Lo consideraremos un "hecho social" (parafraseando a Émile Durkheim) que se presenta en todas nuestras sociedades de manera regular, independiente de otros aspectos. La definición de los delitos en cada nación ha sido determinante en la creación de tipologías sociológicas, pero la interpretación de ellas y de los actores es ciertamente el objeto de la sociología, pues es un proceso de construcción social del crimen y del delincuente. Los comportamientos desviados son en este sentido una construcción colectiva.

En todos los enfoques, las normas son esenciales para definir, describir e interpretar el crimen. Los códigos penales de cada país muestran la influencia de diversos grupos dominantes así como de las profesiones del derecho y de la criminología en la formulación de las normas y valores que las sustentan (como podemos ver en temas que van desde el delincuente juvenil hasta el aborto, su tipificación penal y sus variantes), y en algunos casos por convenciones internacionales (como es el caso de las drogas ilícitas, o los códigos internacionales contra la piratería o las reglas en materia sanitaria). Pero la legislación nunca es ni homogénea ni consistente ni legítima. Por ejemplo, la producción, distribución y consumo de la marihuana en Uruguay está legalizada, mientras que otros países la prohíben. Otro caso controvertido es el aborto, una práctica criminalizada, que solo está permitida en ciertas ciudades (como en la Ciudad de México).

Por lo que se refiere a la noción de violencia en la Sociología, está asociada tanto con la teoría clásica, como en varias disciplinas, y ha sido incorporada como una 
categoría multifactorial, constitutiva del crimen, especialmente desde los años $80^{7}$. Desde entonces, esta está vinculada con la violación y el daño al bienestar individual, personal o de grupo (Misse, 2019, p. 24) y refiere tanto a las agresiones físicas o psicológicas, personales, interpersonales o colectivas, como a otras actividades criminales. Su evolución es concomitante no solo con el desarrollo nacional sino con el surgimiento de un "mercado" global de la misma (Elwert et al., 1999), así como con la creación de las nuevas "redes de crimen global" (Carrión, 2017).

\section{Las dimensiones de la Sociología del Crimen: Temas generales y casos por país ${ }^{8}$}

El conjunto de actos violentos y transgresiones a las normas, muchas de las cuales son ilícitas, es sumamente amplio en nuestros países. Para analizarlos proponemos una clasificación analítica en un conjunto de dimensiones, que incluyen desde conductas violentas interpersonales, como son los crímenes contra las personas, comenzando por el homicidio, y luego contra sus propiedades, tales como asalto, robo, extorsión, secuestro; así también podemos agrupar una serie de actos ilícitos en relaciones de transacción, como la piratería, el fraude, el contrabando o el narcotráfico. Algunas actividades ilícitas requieren de alguna forma de asociación continua, una organización delictiva, entre ellas el tráfico de drogas ilícitas, el tráfico humano, sexual, de órganos, el tráfico de armas, el lavado de dinero o la corrupción. Finalmente hay que tomar en cuenta las respuestas sociales y estatales a la criminalidad, por lo que las policías y los sistemas de castigo serán incluidos en el trabajo.

Para el presente trabajo construimos una clasificación dentro de 16 temas de las distintas corrientes sociológicas en la región. Para ello, tomamos en consideración los ensayos clasificatorios previos (ver: Adorno, 1993, Zaluar, 1999; Barreira y Adorno, 2010; Gabaldón, 2010; Lima y Ratton, 2011; Freitas y Ribeiro, 2013; Imbusch et al., 2011; Campos y Alvarez, 2017; Santos y Barreira, 2016; Briceño, 2016; Ratton, 2018; Misse, 2019; Fellini Fascinetto et al., 2020). Todos ellos cubren las principales conductas delictivas violentas, las transgresiones individuales, colectivas. A partir de esto seleccionamos siete grandes temas:

- La percepción y miedo al delito;

- Las múltiples y diferentes agresiones interpersonales o colectivas cometidas en los

7. Briceño-León (2016) publicó recientemente un trabajo en donde concuerda que la Sociología de la Violencia es un campo emergente, que resuelve problemas no aclarados por la Criminología y la Sociología de la Desviación y que se nutre de otras disciplinas, como la Salud Pública.

8. Una versión previa de esta sección aparecerá en Alvarado (2020). En Oxford Handbook of Sociology of Latin America, section 7: Sociology of Crime. Oxford University Press. 
hogares, en las familias, en los barrios, en las escuelas, en el trabajo o en instituciones y lugares públicos. Estas incluyen las categorías de "delincuencia común" y abarcan desde asalto, lesiones, homicidio, crímenes contra las personas y sus bienes, así como delitos y violencias sexuales (feminicidio) y conductas colectivas (como los linchamientos).

- Los estudios dedicados a las organizaciones criminales o al crimen organizado (Alvarado, 2019a, p. 15), que ocurren en áreas económicas, sociales o políticas. El tema incluye múltiples formas de organizaciones armadas no estatales.

- Violencia y crimen urbanos, regional, fronterizo y otras categorías de la geografía de la violencia en espacios o territorios urbanos (o rurales, pero cada vez menos).

- La sociología de las organizaciones policiales y otros actores coercitivos del estado, como los militares (incluye sus prácticas de corrupción y colusión con el crimen).

- La sociología de la punición, del castigo, de los sistemas carcelarios; la organización dentro de las prisiones.

- Sociología de los jóvenes y la violencia juvenil. La juventud como víctima, como agresora, el primo-delincuente, las organizaciones juveniles y otros temas vinculados, como la muerte violenta, las abducciones y tráfico de personas.

$\mathrm{Al}$ seleccionar estos temas tomamos en cuenta el repertorio de investigaciones previas así como los resultados que analizamos en la exploración bibliográfica. Las búsquedas arrojaron un conjunto de temas en donde se registra más literatura. También lo comparamos con otros estudios temáticos de la Sociología de la violencia y con el desarrollo de tópicos de la Criminología, de la Salud Pública o la Ciencia Política9?. Adicionalmente, de acuerdo con un ensayo de Briceño (2016), este campo de Sociología de la violencia se ha construido al ampliar y profundizar sobre viejos temas e incluir nuevos. Por ejemplo, los estudios de los homicidios han destacado la enorme magnitud de hombres agredidos y víctimas así como el creciente impacto de las armas de fuego. También se ahonda en la importancia de las pandillas en los comportamientos delictivos de los jóvenes, en el sentimiento de miedo, en la violencia urbana en las ciudades y territorios como fronteras (la pobreza urbana y la violencia estructural), en el crimen organizado y en el estado; la policía; la justicia por mano propia y la democracia (Briceño, 2016). Aún más, como lo argumentamos en este trabajo, la Sociología ha empezado a desarrollar sus propias orientaciones

9. Los temas que la Sociología comparte con la Criminología son: (i) control social y político; (ii) policía; (iii) perfil y causas del delito y del delincuente; (iv) las conductas antisociales, (v) prisiones y sistema penal, y (vi) violencia del estado y no estatal. Ahora bien, no comparte plenamente el estudio de los sistemas penales y del poder, de las políticas públicas y de la prevención del delito, entre otros (Gabaldón, 2010 , pp. 256 y 257 ). 
y explicaciones de las policías, los sistemas carcelarios y los penales, de manera que podríamos decir que el desenvolvimiento de la disciplina ha generado sus propias identidades y explicaciones, alternativas a otras disciplinas. Existen muchos otros temas (diez más) que no analizaremos aquí por razones de espacio y porque su desarrollo es desigual y mezcla varios campos disciplinarios. Entre ellos están la violencia política e institucional del estado, que entabla diálogos entre Sociólogos y Politólogos y junto con esto la criminalización de los conflictos y movimientos sociales o el control político de grupos que se oponen a gobiernos autoritarios, que incluye la violencia institucional, estructural (ver por ejemplo Glaucio Dillon, 1993, y sus trabajos sobre la dictadura militar y la represión con Celina D’Araujo, 1994) o la procuración de justicia. También hay producción importante en violaciones a los derechos humanos y a las leyes cometidas por parte de agentes del estado (policías, fiscales, las fuerzas armadas, los celadores - Pinheiro, 1984). Abarca desde las prácticas no reguladas de detenciones, como el arresto arbitrario, llegando a formas graves como la desaparición forzada, las ejecuciones extrajudiciales y otras formas de letalidad policial y militar. Otros temas centrales importantes en la subdisciplina son la violencia juvenil (Alvarado y Tenenbaum, 2020), el efecto del crimen sobre las víctimas, el desplazamiento forzado, las desapariciones, las masacres, la violencia basada en el género, los feminicidios, el cibercrimen, el crimen ambiental, el tráfico de armas, tráfico de personas, de especies, de niños, el tráfico de órganos o el lavado de dinero. Tampoco analizaremos la literatura sobre las políticas públicas, como la prevención del delito, o sobre la emergente producción de narcotráfico, organizaciones criminales y guerras, que tienen una fuerte influencia politológica. La creciente literatura sobre estas agresiones explora estas prácticas en sus dimensiones objetivas y subjetivas, así como en enfoques micro y macro, con múltiples estrategias metodológicas ${ }^{10}$.

10. Algunos expertos argumentan que la Sociología del Crimen en la región tiene influencias no solo de la teoría clásica, sino del psicoanálisis, de la teoría de la acción racional, de la escuela de Chicago, de la escuela de Frankfurt, de Durkheim, de la teoría de Talcott Parsons, Robert Merton, de los estudios experimentales del curso de vida; de los estudios fenomenológicos, de los procesos civilizatorios de Elias; de los enfoques violencia de Arendt; de las orientaciones de la microsociología y de la etnometodología de Erving Goffman. Además algunos argumentan que la disciplina ha avanzado emulando estudios y temas de otras regiones y no sus propios paradigmas (De Freitas y Ribeiro, 2013, p. 87). Cierto es que podemos encontrar varios ejemplos que replican o reproducen parcial o totalmente estas escuelas y sus autores, sobre todo en la segunda etapa de desarrollo de la Sociología. Pero a medida que aumenta la producción sociológica podría argumentarse que sus avances no residen en las réplicas de teorías, sino en el trabajo empírico y en el avance metodológico y en la crítica de las teorías centrales. 
Interpretación y explicación de los fenómenos emergentes del crimen (sub.)

La interpretación y explicación del crimen ha sido abordado desde muy diferentes enfoques teóricos, desde los paradigmas clásicos, hasta los ensayos teóricos innovadores. Los primeros estudios sobre la delincuencia tuvieron influencia de la criminología y un enfoque centrado fundamentalmente en el individuo y la personalidad delictiva. Entre las primeras exploraciones había ensayos que intentaban explicar el acto delictivo, pero también preguntas más amplias como: ¿qué explica que América Latina sea un subcontinente tan violento? (Dillon, 1993, y Dillon, 2011, en Lima y Ratton, 2011). Otros acercamientos exploraron el problema del control social y el comportamiento desviado. Posteriormente, la investigación examinó las relaciones entre la delincuencia y otras variables demográfico-sociales, tales como la edad, la clase social, la raza, la juventud, así como en dimensiones macro-sociales como la desigualdad, la pobreza, el empleo, la educación y en otros grandes procesos, como la urbanización, el cambio social y económico (la industrialización), el control y la integración social. No existe el día de hoy un paradigma dominante en la región, lo cual ilustre el debate dinámico, el desenvolvimiento de la disciplina y su dispersión, y eso nos muestra las insuficiencias, la necesidad de mayor reflexión analítica. Numerosos actos violentos ocurridos en la región no han sido abordados ni explicados por la Sociología, tales como los feminicidios o la nueva violencia política, las desapariciones forzadas, o la utilización de técnicas terroristas tanto por parte de actores criminales como también los estatales. A pesar de los avances en la disciplina, Sandoval (2014, p. 102) argumenta que la Sociología no ha logrado ofrecer teorías e interpretaciones sobre muchos crímenes violentos o actos de barbarie ocurridos en la región, como las masacres, el genocidio político, el asesinato selectivo, el secuestro, la utilización de la crueldad para producir la muerte o la violencia contra las mujeres por parte de actores en conflicto.

Selección de casos y periodización. Tres etapas del desarrollo histórico de la Sociología del crimen

Para la selección y clasificación de los casos consideramos cuatro aspectos: en primer lugar, la formulación y difusión de investigaciones sobre el crimen con base en la teoría sociológica; en segundo lugar, la creación de una comunidad científica de expertos, en tercer lugar, una infraestructura (instituciones, financiamiento, publicaciones), y, en cuarto lugar, por supuesto, el desenvolvimiento del "hecho" delictivo violento.

En este trabajo clasificamos la literatura en tres períodos de progreso del campo: anotamos el origen junto con la primera generación de estudiosos que provienen de la Criminología, la Psicología o de la Sociología del Conflicto o de la Conflicti- 
vidad. Este período consiste en la germinación de los estudios empíricos, alrededor de la década de 1960. A continuación, encontramos un segundo período de desenvolvimiento y formación de subtemas, de la consolidación de una comunidad de expertos que aumenta su tamaño y su densidad (incluye la formación de núcleos de estudio, laboratorios, la creación de carreras profesionales en universidades y nuevas reglas laborales, el apoyo a tesis y los congresos) y de la ampliación de escuelas de Sociología. Este período cubre aproximadamente desde la década de 1970 hasta fin de siglo. En esos años las orientaciones de la Sociología del Conflicto y la Marxista tuvieron auge hegemónico. Aun así, los golpes militares en varios países del cono sur, como el de Argentina en 1976, cortan esta corriente y desautorizan o desmantelan a las comunidades. En muchos casos el desarrollo se trunca ${ }^{11}$. Al terminar este período surgen algunas interpretaciones macro-sociológicas, vinculadas con la transición, como las preocupaciones por la inseguridad, por el anterior terrorismo de los aparatos del estado. Concurre también una crisis del pensamiento marxista y la valoración crítica de los movimientos revolucionarios en la región. La sociología se diversifica en las universidades públicas y la interpretación de las transiciones a la democracia se torna tema hegemónico, junto con la instauración del estado de derecho, los derechos humanos, la percepción de inseguridad, la búsqueda de modelos de seguridad ciudadana así como otros temas clásicos, como la relación entre marginalidad y urbanización (Campos, 1978), la emergencia de la violencia urbana (Briceño, 2002), y el aumento de los homicidios. De nueva cuenta se revisan enfoques teóricos originados en países del hemisferio norte, que al final del período permitirán la diversificación teórica y metodológica. En la gran mayoría de los países los procesos de democratización están acompañados de ajustes y recesiones económicas profundas. $Y$ el tercer período abarca desde el inicio del siglo hasta nuestros años, que consiste en una expansión de todas las dimensiones señaladas: un mayor número de comunidades de conocimiento, más densidad; una nueva competencia entre grupos y "paradigmas", más financiamiento institucional (y mayor estabilidad de las agencias), un incremento exponencial de publicaciones, una suerte de expansión y dispersión temática. Es el momento de consolidación del campo, aun cuando todavía no tiene un anclaje analítico común que lo articule, sino una serie de temas empíricos en expansión.

11. En los cruentos años de la dictadura emigraron de sus países importantes grupos de intelectuales, académicos y hasta escuelas, que eran expulsados de sus países, produciendo una semilla de diálogos y controversias contra las dictaduras y promoviendo las ciencias sociales en otros países. 
Primer período: Los orígenes y desarrollos nacionales de la explicación del crimen en un conjunto de países

Colombia

El primer ejemplo del período y de los casos nacionales es Colombia, donde la dinámica política y los conflictos civiles indujeron a que estudiar la violencia se tornara un tema en sí mismo, autónomo. Los enfrentamientos civiles, las guerras, la proliferación de organizaciones armadas forzaron a los investigadores a ensayar explicaciones. Posteriormente, el desenvolvimiento de la economía de la hoja de coca y sus derivados atrajo expertos y generó una nueva vertiente de estudios. Estos eventos llevaron a la formación de una especialidad que en el país bautizaron como la violentología, "la única rama de la Sociología específicamente colombiana", que paradójicamente no tiene un previo desarrollo sólido de su teoría (Cubides, 1998. Y también Sánchez, 2013).

En Colombia surgió el primer trabajo completo e interdisciplinario sobre el tema de nuestro tiempo, La Violencia en Colombia, escrito por Guzmán, Umaña y Fals Borda en 1958 (publicado en 1962). Promovido por la presidencia de Colombia, este abarcador y precursor reporte exploró las causas e interpretaciones de la prolongada violencia en el país, no solo el conflicto rural (Barreira, 1992) sino la violencia urbana (Camacho y Guzmán, 1990). A partir de entonces, cuatro temas marcaron las rutas de los trabajos colombianos. En primer lugar, el continuado proceso histórico de los conflictos, las consecuencias sociales de la guerra, la violencia política y homicida (que asume distintos componentes: los asesinatos políticos; los conflictos civiles armados, las organizaciones y los actores. Ver Pecaut, 1987. Cataño y Restrepo, 1989). Derivado de esto encontramos el segundo tema sobre violencia homicida, en donde se han producido numerosos aportes multidisciplinarios (por ejemplo, los trabajos de Camacho y Guzmán, 1990; de Espitia, Guerrero, y Concha-Eastman para Cali, 1994. El de Franco de 1997, Ortiz, 1998, y Londoño y Guerrero, 2000, y Vizcaíno, 2002). El tercer tema es la droga en Colombia (particularmente la cocaína) que se tornaría el tema más difundido en la región y a nivel internacional (encontramos 2.644 registros de publicaciones desde el año 2000 en castellano). El cuarto tema está asociado con los procesos de paz contemporáneos y sus complicaciones, los desarmes, los reacomodos institucionales y la recomposición de grupos armados. Entretejidos con estas grandes vertientes de trabajo, se realizaron numerosas investigaciones sobre los temas de homicidios, jóvenes, bandas, organizaciones criminales, víctimas, victimarios, desapariciones, desplazamientos forzados, masculinidades violentas, entre otros. Por ejemplo, sobre la violencia urbana está el trabajo de Concha-Eastman etal. 
(1994) y sobre la policía (Camacho, 1994b), Restrepo et al. (1994), Franco (1999), Fals Borda (2001); sobre violencia organizada, Suárez (1999). Varios trabajos de Colombia y otros países sobre el tema aparecieron en Rotker (2000).

Brasil

El primer trabajo de este campo en Brasil lo publicó Michel Misse con su estudio sobre la delincuencia juvenil en la bahía de Guanabara (1973). Desde entonces se gestó la primera generación de sociólogos estudiosos de la conflictividad, la delincuencia o la violencia, que partieron de los paradigmas del conflicto y fueron abordando nuevos temas, como las consecuencias de grandes transformaciones urbanas y sociales, la marginalidad y las consecuencias de la "modernización”, el sistema de justicia criminal, la policía y las prisiones ${ }^{12}$. Algunos estudios bibliográficos de Roberto Kant (2000), Cesar Barreira y Sergio Adorno (2010), De Freitas y Ribeiro (2013, p. 87) documentan la rápida expansión de la investigación en su país. Imbusch, Misse y Carrión hicieron una compilación más reciente (2011). Barreira y Adorno estiman que el período de 1980 a 2013 (33 años) se publicaron 2.200 artículos, en temas que incluyen desde los estudios del delincuente individual y juvenil (el sujeto "criminoso" o la figura del "bandido" - Misse y Motta, 1979) así como publicaron los primeros estudios de homicidios y, sobre todo, la muerte violenta producida por las policías. Sobre este tema se publicaron, entre otros trabajos, los de Pinheiro et al. (1991); Barcellos (1992); Mingardi (1992); Huggings y Mesquita (1995); Cano (1997) y Cano y Fragoso (1997); Waiselfisz (1998). También exploraron las instituciones del sistema de procuración de justicia, el comportamiento policial, las prisiones y las políticas de seguridad pública. Así, por ejemplo, están las primeras publicaciones de Edmundo Campos sobre criminalización de la marginación $(1978)^{13}$, como también un trabajo de Misse y Motta (1979) y otro de Piquet (2000) sobre crimen urbano. Igualmente, existe un trabajo sobre linchamientos y violencia rural de Almeida (1997) en un interesante libro sobre Sociología Jurídica y justicia privada (Andrade, 1997). Sobre la policía, entre los primeros trabajos están uno de Antônio Luiz Paixão, (1982a y 1982b); otro de Chevigny et al. (1987); Datafolha

12. La primera generación de investigadores académicos en Brasil incluye Alba Zaluar; Antônio Luiz Paixão; César Barreira; Claudio Beato; Edmundo Campos Coelho; Gláucio Soares; José Vicente Tavares dos Santos; Julita Lemgruber (1983); Luciano de Oliveira; Luiz Antonio Machado da Silva (2004); Luiz Eduardo Soares; Maria Stela Grossi Porto; Michel Misse; Paulo Sérgio Pinheiro; Roberto Kant de Lima; Sérgio Adorno, entre otros (algunas personas impulsaron el desarrollo de los trabajos, como Elizabeth Leeds, quien ha sido directora de la oficina de Fundación Ford en Brasil). Fellini Fascinetto et al. 2020 ofrecen. un panorama de esta generación y sus "linajes", que crearon sucesivas cohortes de especialistas.

13. Campos publicó un libro sobre militares en 1985. 
(1995); Muniz et al. (1997); Rifiotis (1999); Caldeira y Holston (1999); Caldeira (2002). Asimismo, Alba Zaluar publicó en 1985 un primer libro sobre la relación entre conflicto y violencia, que fue seguido por varios trabajos de la autora sobre los conflictos y problemas del cambio (1999). Por otro lado, numerosos estudios exploran el tema de drogas (Misse, 1997; Zaluar, 2000) y las formas de organización juvenil y las bandas (Abramovay et al., 1999; Zaluar, 1999), y sobre prisiones (Dias, 2012; Biondi, 2015; Dias y Paes, 2018; Dias y Feltrán 2018).

Argentina

Argentina tiene una larga trayectoria de aportaciones en Sociología y Criminología y tiene un claro corte alrededor del golpe militar de 1976. La literatura previa al golpe era amplia y con fuerte contenido criminológico, como el libro de Cuneo (1970) sobre las Cárceles, o de Nosiglia y Juárez (1986) sobre el delito.

Una manera de trazar el crecimiento de la literatura en el período post-dictatorial es siguiendo el desarrollo de algunas publicaciones, entre las que destaca la revista Delito y Sociedad, la de mayor presencia regional en el tema. Al iniciar sus entregas en 1992, Juan Pegoraro, el primer editor, propuso construir el tema de "lo delictual". Y el background teórico que apoyaba estas ideas quedó plasmado en el primer número con una cita de Foucault (Pegoraro, 1992, p. 1), donde se consideraba que el trabajo filosófico era el fundamento de la nueva Criminología. Publicaciones de esos años exploraron la delincuencia desde la Criminología y la Sociología internándose en interpretaciones de las conductas "irregulares", de la condición delictiva de los adolescentes y el papel de las cortes. Otra influencia de esos trabajos provino de las ideas marxistas asociadas con la lucha de clases y las funciones del estado. Entonces hicieron presencia los trabajos sobre instituciones del estado, como las policías, los militares, o el sistema judicial como instrumentos opresores de las clases dominantes. Emergían las categorías de "inseguridad" y de "control social” o percepción de inseguridad, que indicaban estrategias de naturalización o normalización de conductas por las fuerzas sociales dominantes (Pegoraro, 1997, p. 53). Dicha revista presentó reseñas de numerosos libros sobre policía, control social y crímenes en Argentina, destacando entre los primeros un dossier sobre drogas con trabajos de Masur (1994); uno sobre sobre empresarios ilegales en Colombia, de Camacho (1994a), otro de Rosa Del Olmo que corresponde con su estudio previo sobre la política de drogas, de $(1992)^{14}$, y uno más sobre la selectividad en los arrestos y la discreción policial,

14. La autora venezolana publicó en 1980 un libro sobre los grupos criminales asociados al dictador de Nicaragua y en 1990 otro sobre violencia policial en Venezuela. 
de Gentilli (1995). La producción de estudios sobre el delito y el delincuente, sobre la violencia urbana, la violencia política, sobre policías y jóvenes. Para proporcionar algunos ejemplos tenemos varios estudios sobre policías de Saín (2002), Hinton (2006); otros sobre jóvenes y trafico de niños, de Mura (1997), sobre las mafias (Zinni, 1992), sobre violencia juvenil, como el de González (2004); algunos estudios sobre las violaciones a derechos humanos durante la dictadura (Crenzel, 2008) y un trabajo de Marín reflexionando sobre los hechos armados (1996).

México

México tiene un desarrollo disciplinar relativamente más lento y tardío. Los primeros estudios fueron influidos por la Criminología, la Psicología y la Psiquiatría de la época, así como por un ambiente de conflictos de dimensiones menores si las comparamos con lo que ocurría en Colombia y Brasil. Las preocupaciones estaban asociadas con las consecuencias no deseadas del desarrollo, de los grandes cambios sociales, de la modernización, que proponía que los delitos desaparecerían con el progreso y la urbanización. Un ejemplo temprano fue El costo social del delito, de Quiroz y Quiroz (1970). Al llegar la década de los 1980s, aparecieron los primeros trabajos con nuevos tópicos, como el trabajo sobre la policía de Nacif (1986) y de Botello et al. (1998); el de Nelligan (1988) sobre homicidios de mujeres, los cuales se multiplicaron en la década de los noventa, como el de Johns sobre violencia (1995), de Azaola y Yacamán (1996) sobre mujeres en cárceles, y algunos sobre policía, de Schmid (1996), Arteaga y López (1998) y de Martínez (1999). Rafael Ruíz publicó su libro sobre el crimen urbano, Criminalidady mal gobierno (1998), en donde mostraba la influencia de las escuelas francesa y norteamericana. Además, el desarrollo de las carreras de Sociología y sus posgrados en los años 1970, así como un importante influjo de nuevas generaciones de mexicanos y otro apreciable ingreso de refugiados del cono sur, permitieron la consolidación de las primeras generaciones de "científicos sociales". Poco años después el Conacyt (fundado en 1970) impulsaría la investigación social (lo que contrasta con el apoyo temprano a las ciencias en Brasil, con el Conselho Nacional de Pesquisas de Brasil (CNPq) que data de 1951, el Conicet de 1958 y el Colciencias de 1968).

¿Cuál es el balance del período de inicio de la investigación social en violencia y crimen? ¿Cuáles son las principales influencias teóricas en el primer período? No había una orientación dominante. Tampoco surgió una teoría local. La magnitud y desenvolvimiento empírico e histórico de los temas parecían dominar la reflexión analítica, que recurría o reproducía teorías de origen norteamericano o europeo. En este período se retomaron varias orientaciones sociológicas clásicas y otras más 
novedosas. Por ejemplo, algunos trabajos emplearon el enfoque de Merton sobre desviación social, anomia y tensión social para analizar los hechos delictivos y el comportamiento desviado de las normas sociales. También usaron los enfoques de E. Durkheim y de adherentes al funcionalismo o a las teorías de la modernización. Pero también hubo influencias de Howard Becker en la construcción del acto desviado (1963, pp. 8-9). Freitas y Ribeiro (2013) hicieron una taxonomía de las variadas concepciones y sus autores utilizados en los estudios brasileños (anexo 3: 112-113). Constatan que muchos trabajos se realizaron emulando las teorías clásicas. Otro resultado fue el incremento de la sensación de miedo, que tenía una clara influencia de la Psicología, así como de la percepción de inseguridad, que estaba más anclado en la Sociología. Originalmente estas dos categorías estaban vinculadas con el temor a las acciones opresivas de los gobiernos autoritarios. En consecuencia, floreció el tema de la represión de la autoridad y al acoso policial tanto en Brasil (Fausto, 1984; Dillon, 2011, y D’Araujo, 1994) como en Argentina (Kessler, 2009; Kessler y Otamendi, 2020). Pero en el caso de México sus raíces provienen de la percepción de inseguridad urbana, el miedo al delito y a otros riesgos de la vida urbana contemporánea (Reguillo, 2000). Estos temas no tuvieron una base displinaria común, como son los casos de los estudios policiales. También los estudios carcelarios o los de jóvenes mezclaban orientaciones jurídicas, criminológicas o políticas. Los estudios urbanos empezaban a cobrar fuerza, combinando la geografía, la planeación, la economía y la sociología.

Tal vez una de las mayores y más duraderas influencias después del período marxista y del retorno a las democracias en Brasil y Argentina fue Michel Foucault sumada a algunas corrientes criminológicas nuevas. Fue en estos países en donde se forjaron novedosas explicaciones críticas del rol del estado (y de sus políticas públicas). Pero en México no encontramos una corriente o un autor dominante.

Segundo período. El cambio estructural en la región, la expansión de la sociología del crimen y de la violencia y la consolidación del campo

En la década de los años ochenta los expertos comenzaron a utilizar un rango más amplio de teorías y metodologías para explicar tanto los problemas originales como muchos otros temas emergentes. Fue la era del retorno de gobiernos democráticos, cuando los estudios retomaron una nueva dinámica tanto nacional como comparativa y tuvieron que responder a las modalidades de violencia criminal emergentes. Había entonces una mejor disponibilidad de información pública. Este fue un período de cambios profundos en las economías, en las políticas públicas nacionales, en el tamaño de los gobiernos y en las capacidades de los estados. Los gobiernos intentaron superar el estancamiento económico promoviendo la desregulación, la reducción 
del gasto social, una creciente precarización del trabajo, todo lo cual intensificó la desigualdad y restringió la estructura de oportunidades para las poblaciones más necesitadas. La democratización estuvo acompañada con estos cambios y sobre todo con un incremento sostenido de todos los tipos de comportamientos delictivos "comunes" (UNDP, 2010). Estos son indicadores de una transición estructural en la región. Hay un amplio consenso de que la situación empeoró, lo que obligó a los estudiosos a identificar las condiciones y causas de esta nueva era de patrones de “sociabilidad violenta" (Machado, 2004, p. 55).

Hay que destacar que durante la democratización emergieron dos enfoques sobre la inseguridad que terminaron por considerarse como paradigmas construidos y compartidos entre los expertos de la región: la Seguridad Ciudadana y una paralela de Seguridad Humana. Marcaron una nueva posición crítica del debate político e ideológico sobre el crimen y estaban sustentados en la demanda de implementar los derechos humanos. Estos paradigmas tienen como centro de su trabajo los derechos ciudadanos y la idea de la coproducción de la seguridad entre los ciudadanos y el estado, lo que impuso nuevos retos a los regímenes emergentes en la construcción de una sociedad justa, igual y legítima (Tulchin, 2003) y que demandaba investigaciones sobre la violación a los derechos humanos durante las dictaduras. Estos trabajos resultaron en una rica veta de producción, comenzando con los trabajos de Rico y Chinchilla (2002), Frühling et al. (2004), Bailey y Dammert (2006), entre otros. Junto con estas intervenciones surgió una nueva literatura sobre inseguridad, corrupción e impunidad (Davis y Alvarado, 1999), sobre abuso y letalidad policial, y sobre violaciones a los derechos humanos; entre ellos, Cano (1997); Lima R. K. (1999); Cano y Santos (2001); Costa y Stepan (2001); Saín (2002); Gómez et al. (2003); Frühling et al. (2005); y después Cano y Duarte (2012). También emergieron trabajos sobre reformas policiales (Tiscornia, 1997; Soares, 2000; Sain, 2002; Mesquita, 2004; Frederic, 2008; Gabaldón y Antillano, 2007), sobre todo los de orientación comunitaria (Mesquita Neto, 2004; Fontecilla y Suárez, 2014; Adorno, 2019; Alvarado, 2019b), sobre las relaciones entre la policía y los jóvenes (Zavaleta et al., 2016), y sobre violencia policial y urbana (Caldeira, 2000; Salla, 2001; Willis, 2013; Pérez et al., 2018; Hathazy y Frederic, 2018) y sobre militarización de la seguridad (Alvarado y Zaverucha, 2010; Jiménez y Turizo, 2011, p. 113).

En el segundo período, destacan dos trabajos en Colombia: Colombia: Violencia y Democracia, coordinado por Gónzalo Sánchez (1987), y el de Daniel Pécaut, Orden y violencia en Colombia (1987). El primero es producto de una comisión de diez investigadores, cinco sociólogos, analizando los actores, las modalidades de la violencia de diversos protagonistas y la pluralidad de víctimas, en donde postularon nuevas categorías de violencia. 
En México al cierre del siglo xx apareció la preocupación por el tráfico de drogas ilícitas, con trabajos de Bagley (1997), Chabat (1997), Arzt (1998) y sobre las organizaciones y su cultura (Astorga, 1995 y 1997). Además, iniciaron los estudios sobre inseguridad y las consecuencias del cambio político (Reguillo, 2000). Davis y Alvarado (1999) empezaron sus trabajos sobre inseguridad, policías y cambio de régimen. Dos textos sintetizan las preocupaciones de los expertos en Argentina. El de Tiscornia (1997), sobre la Violencia policial y otros sobre la represión militar (Hershberg y Agüero, 2005).

Tercer período: la búsqueda de nuevos paradigmas, la diversificación de los estudios empíricos y la fragmentación académica

Este período es el de mayor expansión del campo, con nuevas generaciones de investigadores formados en las escuelas de la región, con apoyos para financiar la educación y la investigación. Se acompaña también de dos grandes procesos políticos y criminales. Por un lado, en contraste con las expectativas, los regímenes políticos no consolidaron un régimen democrático de derecho y mantuvieron bajos los indicadores de gobernanza, con la persistencia de una enorme desigualdad, corrupción generalizada, una creciente violencia organizada, una alta impunidad y ausencia del control de la coerción pública, entre otras cosas. Por otra parte importante, la violencia tuvo un incremento inexorable en la región, así como en escala transnacional, durante el período de auge de grandes firmas criminales.

Una consecuencia dramática del estado de cosas descrito es que los ciudadanos vivan en una condición de desprotección y precariedad de sus vidas y sus derechos por décadas, desde la violencia en sus barrios hasta ciudades bajo control de organizaciones criminales (Briceño-León y Camardiel, 2015). Viven en zonas con las más altas tasas de homicidios del mundo (wHO, 2014; Alvarado et al., 2015), las más altas tasas de feminicidios y de homicidios de jóvenes (Concha-Eastman, 2015). Sus comunidades tienen los mayores mercados de armas de fuego. En algunas ciudades son víctimas de las batallas entre organizaciones armadas no estatales y han sido testigos que, en algunos casos, los jefes criminales llegan a formar parte de la élite política y someten a los habitantes a una servidumbre sin ley.

Hoy día en el subcontinente las numerosas organizaciones criminales presentes no solo desarrollan guerras entre ellas; también atacan a los ciudadanos indefensos, amenazan o compran a sus gobiernos. Para ahondar en los problemas, las organizaciones coercitivas de los estados (la policía y los militares), siguen perpetrando detenciones arbitrarias, tortura y ejecuciones extrajudiciales, crímenes contra mujeres, así como permanecen algunos escuadrones de la muerte. 
Es el período de cristalización o de mayor ostentación del poder económico y político adquirido por las organizaciones criminales, por su organización industrial, corporativa transnacional, que se acompaña del declive y fragmentación del poder de los estados. Es la era de los paramilitares, las milicias, las nuevas pandillas trasnacionales, las autodefensas, los combos, y la transfiguración de viejas organizaciones guerrilleras, que controlan territorios enteros dentro de los países y limitan la soberanía estatal. Los actores criminales cambian su relación con los estados y con la sociedad, con las clases sociales y con las élites; han creado nuevas relaciones sociales, económicas y una nueva gobernanza.

De acuerdo con Adorno y Salla (2007), la emergencia de las organizaciones criminales no puede separarse de las cambiantes condiciones sociales, particularmente después de las reformas económicas que desregularon los mercados y alteraron los controles nacionales y fronterizos. Aún más, motivaron la movilidad de capitales sin reglas, creando condiciones favorables para los flujos ilícitos tales como el tráfico industrial de drogas, de personas, de órganos humanos, de armas, para el contrabando, el fraude fiscal y el lavado de dinero (actividades que hoy días son globales y utilizan las redes bancarias y otras corporaciones). También podría argumentarse que la urbanización acelerada y sin reglas produjo condiciones de precariedad favorables para las actividades ilícitas que, a su vez, reforzaron la segregación y la desigualdad.

En esta etapa reciente se han formulado y explorado nuevas tesis y varios paradigmas que intentan explicar las tendencias criminales individuales, colectivas, urbanas, y que además están acompañadas con investigación empírica más sofisticada. El rango de temas y enfoques hoy día es mucho más variado y fragmentado. Un grupo creciente de expertos ha estado explorando la relación entre integración social, cohesión social y ha examinado políticas que podrían contribuir a reducir el crimen. En este campo varios autores han estudiado la violencia juvenil, la violencia contra la mujer y el feminicidio (como el trabajo de Luz Méndez en Guatemala, 2010 y 2013, o el de Monarrez y Tabuenca en México, 2007) mientras que otros se han enfocado en las teorías del control social o de la eficacia colectiva,-con enfoques sustentados en la sociología de la acción racional, en los paradigmas de las oportunidades criminales o en la privación relativa.

Un texto que sintetiza las preocupaciones de los expertos colombianos en este período es el informe del Centro Nacional de Memoria Histórica titulado ;Basta ya! Colombia: memorias de guerra y dignidad, dirigido por Gonzalo Sánchez (2013). Este tercer período es de un ambiente generalizado de violencia en algunas regiones y de países con territorios enteros bajo control de organizaciones criminales y fuera del estado. Por ejemplo en México el territorio de "tierra caliente" de los estados de Michoacán y Guerrero ha estado en disputa entre organizaciones criminales 
(Maldonado, 2010; Fuentes, 2015), mientras que el Cártel del Golfo y posteriormente los Zetas controlaron buena parte del Golfo-norte del país (Correa, 2017). Situaciones parecidas ocurren en Colombia con los territorios donde han operado las guerrillas como las Farc (Villamizar, 2017), en ciudades de la región como São Paulo, donde opera el PCC (Dias, 2013; Alvarez, 2013; Paes y Dias, 2018; Amorim hizo un trabajo de análisis de dos organizaciones criminales en Rio y en São Paulo, 2003), y en ciudades de Centroamérica donde las maras controlan buena parte de los barrios populares.

Cada país ha tenido que responder a problemas de violencia nacionales y regionales, y esto tal vez ha contribuido a la continua dispersión analítica. Para terminar, es pertinente señalar el avance intermitente de las ciencias sociales en los cuatro países, el crecimiento desigual y heterogéneo de sus comunidades científicas, así como de las temáticas exploradas. Pero en general se muestra un amplio desarrollo de las nuevas generaciones de estudiosos con herramientas metodológicas más sofisticadas ${ }^{15}$.

\section{Síntesis de las orientaciones y temas}

El tercer período muestra la expansión de los temas y las orientaciones teóricas, así como un sofisticado desarrollo metodológico. Podría hablarse de dispersión, pero prefiero mostrar la fragmentación de los estudios, dado que si bien cada dimensión se ha desarrollado extensiva y exhaustivamente, no hay una articulación entre temas y mucho menos una síntesis del campo, como tampoco hay consensos entre las comunidades epistémicas dentro de los países. Esta sección retoma en forma sintética las siete dimensiones anotadas al principio: las diferentes interpretaciones generadas desde los inicios de la subdisciplina hasta el período actual.

Cada uno de los temas contiene una visión que agrupa varios paradigmas o teorías pero sobre todo utiliza muy distintos métodos, desde las etnografías y los estudios de caso hasta los estudios comparativos transnacionales o los de panel. Dado que el crimen no puede ser explicado por una sola causa, los esfuerzos se han dirigido hacia estudios multivariados y a estrategias multinivel y complementarias de explicación. Algunos autores han realizado encuestas nacionales o entre países, como las de victimización (ver Manzano et al., 2020). Ellos intentan resolver varios desafíos para el avance del campo, entre ellos produciendo datos de calidad y series de tiempo. Otro ejemplo notable de un estudio complejo y ambicioso es el trabajo de

15. Este trabajo muestra el desenvolvimiento heterogéneo y plural de las comunidades de los cuatro países. Entre líneas se pueden ver las aportaciones de otros expertos y colegas de países como Venezuela, Ecuador o Centroamérica, en donde las condiciones no han permitido el mismo fortalecimiento institucional que impulse a las ciencias. El resultado es un desarrollo desigual de sus comunidades. 
Adorno y otros en la Universidad de Sao Paulo durante 12 años, sobre socialización legal en Brasil (Núcleo da Estudos da Violência, NEV-UsP. Adorno et al., 2019). No obstante, prevalece lo que entendemos como nacionalismo metodológico, con un número de casos reducido, donde predominan orientaciones que se auto etiquetan como "cualitativas". Ahora procedemos con un balance final de cada tema.

Una primera dimensión es la de percepción y miedo al crimen, que ha atraído numerosos estudiosos, entre ellos el trabajo de Reguillo (2000), o Kessler (2009) sobre el "sentimiento de inseguridad", un concepto que abarca emociones como el miedo y estudia las respuestas de los sujetos sociales contra la inseguridad en su vida cotidiana.

En un principio los investigadores de la región ligaban el miedo al crimen a los actores estatales, debido al pasado autoritario y la represión y no solo por la intensificación de las ofensas o agresiones delictivas. Los trabajos incluían temas como la confianza en las instituciones y en las autoridades, particularmente la policía y el régimen judicial (todos ellos eran percibidos como corruptos, ineficientes y sesgados contra los pobres; Kessler, 2019). Este campo de investigación está dividido en dos áreas. Por una parte, las orientaciones de la Historia, la Antropología y la Sociología dedicadas a los temores o miedos urbanos, políticos y sociales, y otra ligada con criminología anglosajona cuantitativa.

Otro tema que ha sido preocupación general de todas las comunidades es el estudio de la violencia criminal letal, con numerosos enfoques y esfuerzos por explicar las causas y su enorme magnitud. Concha-Eastman, Muñoz, y Santos (2020) hicieron un esfuerzo de síntesis, construyendo un panel para explicar las altas tasas de homicidio en la región (comparada con el resto del mundo), explorar sus causas, ya sean interpersonales, por las organizaciones criminales o por los conflictos. Después de revisar las explicaciones económicas, en salud pública, en enfoques como la desorganización social, así como otras explicaciones estructurales, sociodemográficas o por indicadores económicos como la desigualdad, el desarrollo, se exploraron también otras dimensiones institucionales, como el funcionamiento del sistema de procuración de justicia. Entre sus hallazgos exponen que, a pesar de las mejoras socioeconómicas, la tendencia de homicidios no se ha reducido en la región, lo que contradice las teorías del desarrollo y de modernización. Encontraron que la combinación de variables como desigualdad y desempleo presentan una relación causal positiva, pero su mayor aporte tiene que ver con el efecto institucional del sistema de justicia criminal, que contribuye a los homicidios y a otros tipos de crímenes, entre otras cosas por la inexistencia de un estado de derecho (Briceño redactó una explicación similar en su trabajo de 2015). Un factor de enorme impacto en las lesiones y la letalidad es la accesibilidad de las armas de fuego en mercados ilícitos en 
prácticamente todas las ciudades del subcontinente. Entre 2000 y 2012 en Colombia, $83.7 \%$ de los homicidios contra jóvenes fueron producidos por armas de fuego; en Brasil 76.3\% y en México 55.8 \% (Alvarado et al., 2015, p. 346).

Por lo que respecta al tema de la violencia urbana, este ha ocupado numerosos autores sobre la relación entre los espacios físicos construidos, su configuración social y las formas (conducivas) de la violencia en ciudades de Argentina, Brasil, Colombia, México, Venezuela, y en algunas urbes fronterizas (Caldeira, 2001; Goldstein, 2003; Carrión y Gottsbacher, 2020; Davis, 2020). La literatura comienza desde los años 1960s con la relación entre urbanización, marginalidad y delitos, y se enruta hacia los temas de exclusión (segregación espacial) y de organizaciones ilícitas en redes urbanas. En los años recientes se han desarrollado trabajos sobre la expansión de redes ilícitas urbanas, algunas armadas, el control de los barrios y zonas populares y sus efectos en la gobernanza urbana. Algunos trabajos estudian las reformas urbanas, específicamente aquellas orientadas a reducir las actividades ilícitas con estudios sobre Cali, Medellín, Quito, São Paulo, Río de Janeiro, la Ciudad de México, El Salvador, y mejorar el acceso de los ciudadanos a la urbe.

Un tema adicional tiene que ver con aspectos territoriales de la violencia, un campo de la literatura que está floreciendo y otorga atención a las fronteras nacionales y otros espacios rurales donde predominan actividades ilícitas. Carrión y Gottsbacher (2020) proponen que la violencia en las fronteras tiene su propia lógica. Muestran cómo las reformas económicas y políticas, junto con la revolución tecnológica, han inducido una pérdida de control de territorios y sectores a lo largo de las fronteras (por parte de los estados nacionales). Espacios que han sido ocupados por una red global de crimen (Carrión, 2017).

Por otra parte, los estudios sobre las organizaciones criminales son cada vez más diversos. Este campo muestra el enorme conjunto de actores alrededor del tráfico de drogas, sus aspectos económicos (su impacto en los mercados ilícitos, el lavado de dinero y la corrupción), aspectos logísticos y las relaciones sociales y políticas que generan, particularmente las nuevas relaciones con los gobiernos. Buena parte de los trabajos se concentran en el desenvolvimiento de las organizaciones de tráfico de drogas que comenzó desde los años 1990s. Durán Martínez (2020) muestra cómo se ha expandido el campo de estudios relativo a la producción, distribución y consumo de drogas ilícitas, el tipo de productos, las estrategias de distribución y la diversificación de actividades.

Pero lo que más destaca del tema es la inmensa diversificación de las formas organizacionales (más allá del narcotráfico), que van desde pandillas en vecindarios urbanos de México o Centroamérica (Santacruz et al., 2000; Castillo para México, 2002) hasta redes transnacionales, como la Mara Salvatrucha o la Mara 18 (Rodgers, 
2017; Cruz et al., 2017), hacia formas más complejas y sofisticadas, como el Primeiro Comando da Capital (Dias, 2013; Dias y Paes, 2018; Feltrán, 2018; Biondi, 2015 y 2018), o los paramilitares (Romero, 2007), los combos y las autodefensas en Colombia o las diferentes formas de las organizaciones guerrilleras, como las Farc (Villamizar, 2017), pero también las organizaciones transnacionales como el Cártel de Sinaloa, el de Jalisco Nueva Generación (CJNG) o los Zetas (Correa-Cabrera, 2017), por mencionar los principales. Estas organizaciones controlan territorios donde implantan sus negocios, mantienen un cuasi monopolio de múltiples actividades ilícitas (algunos diversifican sus actividades hacia la extorsión, secuestro o tráfico de migrantes, la explotación de recursos energéticos o de megaproyectos) y para ellos se coluden, compran o enfrentan a las fuerzas coercitivas de los estados. En ocasiones utilizan los gobiernos locales y llegan a crear sistemas de gobernanza criminal informal (Flores, 2013), como también sindicatos y partidos políticos para controlar a los ciudadanos. Estas organizaciones tienen un poder político que se refleja en sus actividades político-electorales. Por ejemplo, algunos criminales financian candidatos y campañas, generan nuevas formas de violencia política desde los de barrios hasta las ciudades (Arias, 2017, pp. 246-247; Briceño y Camardiel, 2015, pp. 15-24). Otro importante fenómeno son las milicias presentes en varias comunidades de Brasil (Cano y Duarte, 2012, entre otros). Hay una nueva literatura sobre organizaciones creadas por comunidades (autodefensas) que surgen formalmente para combatir otras bandas armadas: cuando los gobiernos no los apoyan, son cooptados por criminales o desaparecen (Maldonado, 2010, y Fuentes, 2015). Algunos trabajos se dedican hoy día a discutir las nuevas configuraciones de los estados, que no tienen el monopolio de la fuerza coercitiva.

En el campo de las cárceles, Lembruger (1983), Pinheiro (1983), Adorno (1991), Salla (2001 y 2006), Azaola (1996), Macaulay (2007), Feltrán (2018), Dias (2012) y Dias y Paes (2018), muestran la genuina evolución de estas instituciones carcelarias en la región, así como la necesidad de crear explicaciones adecuadas a las circunstancias regionales, urbanas y sociales de cada sistema en la región.

Por lo que respecta a la Sociología de las policías y de las fuerzas coercitivas de los estados, los trabajos manifiestan la gran variedad, fragmentación y cambios recientes en estas organizaciones estatales. Hay una metamorfosis de las fuerzas coercitivas de los estados. La región ha sido espacio de innumerables reformas policiales en el sector de seguridad, así como fueron hechos experimentos de reorganización de las policías y de la seguridad interna, desde las policías comunitarias hasta la militarización, entre otras cosas, para tratar de reducir abusos y letalidad de las policías.

Entre los temas emergentes está el del desplazamiento forzado. Angarita (2020) revisa el debate intelectual en derechos humanos, que incluye la migración forzada, 
el desplazamiento y los refugiados que sufren violaciones a sus derechos humanos y la emergencia de un perfil social nuevo: la víctima. Describe experiencias de terror, desenraizamiento cultural y desposesión en una región con millones de desplazados.

Otro tema con enorme desarrollo es el de la violencia juvenil, la delincuencia amateur, el empleo de jóvenes por organizaciones criminales o el sicariato, así como la masculinización exacerbada.

En los años recientes hay esfuerzos de investigaciones multinivel para intentar demostrar enfoques norteamericanos, como el de la desorganización social o la eficacia colectiva (Manzano, Mohor y Jiménez, 2020). Otros autores han intentado investigaciones similares, pero no han logrado confirmar las principales hipótesis del paradigma. En síntesis, artículos recientes, como el de Manzano et al. (2020) Concha et al. (2020) o Kessler y Otamendi (2020), entre muchos otros, muestran el incremento en rigor analítico y metodológico para explicar el crimen utilizando de variables institucionales, estructurales, con estudios de paneles y encuestas comparativas transnacionales o con estudios cualitativos. Pero sobre todo expresan los límites de los paradigmas actuales. El avance metodológico en la región ha sido notable, a pesar de la dispersión teórica y de la falta de acuerdos que permitan una síntesis del campo en el futuro inmediato.

Discusión: para una Sociología del crimen

Este trabajo muestra el florecimiento de una comunidad de expertos que emprendieron sus estudios nacionales, con los cuales germinó un nuevo campo de la Sociología del Crimen. Sus logros se manifiestan sesenta años después con el enorme, magnífico incremento de la producción y publicación de literatura plural y una infraestructura consolidada en universidades públicas. Es inverosímil cubrir toda la literatura del campo del crimen en un artículo ${ }^{16}$. No obstante, la genealogía propuesta nos ilustra el florecimiento y los desafíos en la región, en donde hemos intentado interpretar los

16. También es imposible hoy día hacer un recuento de todos los artículos de las revistas latinoamericanas de Sociología o de todos los catálogos de nuestras instituciones. No obstante, destacan algunas con mayor trayectoria en la región, entre ellas: Delito y Sociedad, de Argentina. La Revista Mexicana de Sociología, que curiosamente hasta la mitad de los años 1960s publicó el primer artículo sobre el delito. La Revista Colombiana de Sociologíanació en 1979 y ha tenido varias contribuciones. La Revista Brasileira de Sociología surge en 2013 y tiene algunos trabajos notables (citados aquí). La revista Tempo Social, de la usp fue fundada en 1989, que ha publicado 28 artículos seminales sobre temas de crimen, policía, justicia, cárceles, víctimas y otros temas. Es una revista que publica más artículos sobre estos temas. Precisamente el primer artículo sobre las prisiones es el de Sergio Adorno (1991). Otras revistas han sido vehículo de comunicación plural, Espacio Abierto de Venezuela. La revista Urbio de Ecuador está especializándose en los temas de crimen y más recientemente, La Revista Brasileira de Segurança Pública (2007). 
fenómenos con base en teorías clásicas y contemporáneas, como también exploramos invenciones locales para atender las carencias de las teorías, así como el complejo rompecabezas de varias formas de violencia que se desenredan, crecen, se expanden y abren nuevos campos de forma inquietante. Es un reto y una lucha por comprender y por incidir en un proceso histórico al que no vemos término en el futuro inmediato.

Esta genealogía manifiesta la necesidad de deliberar sobre una posible síntesis del campo, dado que las comunidades han creado una gran cantidad de interpretaciones, fragmentadas entre sus territorios y entre los países. Entre los desafíos de esta Sociología están integrar sus campos y explicar el enorme crecimiento de la sociabilidad violenta.

Quedan muchos tópicos por explorar, como la necesidad en seguir luchando por implementar un estado de derecho (contra las nuevas estrategias de corrupción) y la mayor atención a la violencia basada en el género, y dentro de ella explicar y erradicar los feminicidios o la violencia de género contra mujeres indígenas (Méndez, ibíd.), la revisión crítica del pasado dictatorial (Crenzel, 2008) y la búsqueda de la verdad. Por ejemplo, el desarrollo de los estudios carcelarios nos permite postular que las contribuciones de los autores referidos en el trabajo (Dias, 2012, entre otros) plantean la necesidad de redefinir críticamente las categorías originadas en la literatura de mafias (como las de Europa - en Sicilia, Nápoles, o en Rusia, etc.), porque lo ocurrido en Brasil y en Centroamérica obliga a replantear el origen de estas, sus objetivos formales, su estructura interna y su influencia hacia el exterior, principalmente las sociedades en donde se inserta.

Terminamos con una invitación a estudiar la emergencia de una suerte de nueva estructura social, subyacente a las nuevas formas de trabajo y sociabilidad que emergieron de actividades violentas. En las economías ilícitas encontramos nuevos actores sociales, nuevas sociabilidades y muchas organizaciones emergentes de estas múltiples formas de violencia. Hay nuevos tipos de actores de este mundo criminal, como los jefes de bandas, organizaciones o cárteles. Ellos son empresarios de la violencia, luego están los intermediarios, como los contrabandistas, los grandes traficantes de armas y la piratería. Hay también una nueva clase de políticos asociados con la violencia. Y existe un nuevo ejército de trabajadores de las nuevas formas productivas, los vigilantes (halcones, vigilantes de la clica o la boca de fumo), los transportadores de mercancías (las mulas), los contadores, los cobradores (de extorsiones, de secuestros), los piratas, los expertos en crímenes cibernéticos, los asesinos a sueldo, los pistoleros, los sicarios, hombres y mujeres que asumen nuevos roles de sociabilidades violentas. 


\section{Referencias}

Abramovay, Myriam et al. (1999), Gangues, galeras, chegados e rappers: juventude, violência e cidadania nas cidades da periferia de Brasilia. Rio de Janeiro, Garamond.

Adorno, Sergio (2013), “Democracy in Progress in contemporary Brazil: corruption, organized crime, violence and new paths to the rule of law". International Journal of Criminology and Sociology, 2: 409-425. Disponível em https://nev.prp.usp.br/wp-content/uploads/2015/05/ IJCSV2A38-Adorno.pdf.

Adorno, Sergio. (1996), A gestáo urbana do medo e da insegurança. Violência, crime e justiça penal na sociedade brasileira contemporánea. São Paulo, Universidad de São Paulo.

Adorno, Sergio. (1993), “Criminalidade urbana no Brasil: um recorte temático". Boletim Informativo y Bibliográfico de Ciências Sociais, 35 (1): 3-24.

Adorno, Sergio. (1991), "A prisão sob a ótica de seus protagonistas. Itinerário de uma pesquisa”. Tempo Social, 3 (1-2): 7-40. Disponível em http://www.scielo.br/scielo.php?script=sci_ arttext\&pid=S0103-20701991000100007\&lng=en\&nrm=iso.

Adorno, Sergio \& Salla, Fernando. (2007), "Criminalidade organizada nas prisões e os ataques do PCC”. Estudos Avançados, 21 (61): 7-29. Disponível em http://www.scielo.br/ scielo.php?script=sci_arttext\&pid=S0103-40142007000300002\&lng=en\&nrm=iso.

Adorno, Sergio et al. (2019), “Legitimidade policial: Um modelo de mensuração”. Revista Brasileira de Ciências Sociais, 34 (100): 1-25.

Almeida Alfredo. (1997). "Linchamentos: atos de justiça camponesa entre a humanização da penalidade e a desumanização do indígena”. In: Andrade, Maristela de Paula (ed.). Justiça privada e tribunal. São Luis, UFMA, vol. 2, pp. 85-132.

Alvarez, Marcos et al. (2013), "Das comissões de solidariedade ao primeiro comando da capital em São Paulo". Tempo Social, 25 (1): 61-82.

Alvarado, Arturo. (2019a), “Organizaciones criminales en América Latina: una discusión conceptual y un marco comparativo para su reinterpretación”. Revista Brasileira de Sociologia, 7 (17): 11-32. Disponível em http://www.sbsociologia.com.br/rbsociologia/index. $\mathrm{php} / \mathrm{rbs} /$ article/view/539.

Alvarado, Arturo. (2019b), "Reformas policiales y la construccion de un estado de derecho. El caso de la policia vecinal de Ciudad Netzahualcóyotl”. Desacatos, 60: 34-57. Disponível em https://desacatos.ciesas.edu.mx/index.php/Desacatos/article/view/2089.

Alvarado, Arturo et al. (2015), Vidas truncadas: el exceso de homicidios en la juventud de América Latina 1990-2010. México, El Colegio de México.

Alvarado, Arturo \& Tenenbaum, Gabriel. (2020), "Youth Violence in Latin America”. In: The Oxford Encyclopedia of International Criminology [In press].

Alvarado, Arturo \& Zaverucha, Jorge. (2010), "La actuación de las fuerzas armadas en la seguridad pública en México y Brasil: Una visión comparada”. In: Alvarado, Arturo \& 
Serrano, Mónica. (coords.) Seguridad nacional y seguridad interior. Tomo xv de la serie Los grandes problemas de México. México, Colegio de México.

Amorim, Carlos. (2003), CV-PCC. A irmandade do crime. Rio de Janeiro/São Paulo, Record. ANDrade, Maristela de Paula (org.), (1997), Justiça privada e tribunal. São Luis, UFMA, vol. 2. Angarita, Emilio. (2020), "Forced displacement in Latin America and globalization. Causal and political factors and perspectives". In: Rivera, Liliana \& BADA, Xóchitl. (eds.), The Oxford handbook of the sociology of Latin America. Oxford, Oxford University Press [In press].

ARIAs, Enrique. (2017), Criminal enterprises and governance in Latin America and the Caribbean. Nova York, Cambridge University Press.

Arteaga, Nelson \& López, Adrián. (1998), Policia y corrupción: el caso de un municipio de México. DF, Plaza y Valdés.

ARzT, Sigrid. (1998), PGR y CO y DROGAS. The complexities of a US-Mexican national security threat. Washington DC, WwISC-Program on Latin America.-

Astorga, Luis. (1997), "Los corridos de traficantes de drogas en México y Colombia”. Revista Mexicana de Sociología, 59 (4): 245-261.

Astorga, Luis. (1995), Mitología del "narcotraficante” en México. DF, Plaza y Valdés.

Azaola, Elena. (1996), El delito de ser mujer: hombres y mujeres homicidas en la ciudad de México: historias de vida. DF, Plaza y Valdés.

Azaola, Elena \& YaCAMÁN, Cristina. (1996), Las mujeres olvidadas. Un estudio sobre la situación actual de las cárceles de mujeres en la República Mexicana. México, El Colegio de Mexico.

BAgley, Bruce. (1997), Drug trafficking research in the Americas: an annotated bibliography. us, North-South Center Press.

Bailey, John y Dammert, Lucía. (ed.), (2006), Public security and police reform in the Americas. Pittsburgh, University of Pittsburgh Press.

Barcellos, Caco. (1992), Rota 66: A história da polícia que mata. São Paulo, Brazil. Record. BARreira, Cesar. (1992), Trilhas e atalhos do poder: conflitos sociais no sertão. Rio de Janeiro, Rio Fundo.

Barreira, Cesar \& Adorno, Sergio. (2010), "A violência na sociedade brasileira”. In: BENEDito, Carlos (coord.). Horizontes das ciências sociais no Brasil: sociología. São Paulo, Associação Nacional de Pós-graduação em Ciências Sociais.

BeCKer, Howard. (1963), Outsiders. studies in the sociology of Deviance, EUA. Nova York, Free Press. Biondi, Karina. (2018), Junto e misturado: uma etnografia do PCC. São Paulo, Terceiro Nome. BIOndi, Karina. (2015), Proibido roubar na quebrada. Território, hierarquia e lei do PCC. São Paulo, Terceiro Nome.

Botello, Nelson et al. (1998), Policía y corrupción: el caso de un municipio de México. México, Plaza y Valdés.

Briceño-León, Roberto. (2016), “La sociología de la violencia: un campo nuevo”. Espacio Abierto, 25 (4): 17-30. 
Briceño-León, Roberto. (ed.). (2015), Ciudades de vida y muerte: La ciudad y el pacto social para la contención de la violencia. Caracas, Alfa.

Briceño-León, Roberto. (2002), “La nueva violencia urbana de América Latina”. Sociologías, 4 (8): 34-51. Disponível em https://www.scielo.br/pdf/soc/n8/n8a03.pdf.

Caldeira, Teresa. (2002), “The paradox of police violence in democratic Brazil”. Ethnography, $3(3): 235-63$.

Caldeira, Teresa. (2001), City of walls, California, UCP. California, University of California Press.

Caldeira, Teresa. (2000), Cidade de muros: crime, segregação e cidadania em São Paulo. São Paulo, Editora 34/Edusp.

Caldeira, Teresa \& Holston, James. (1999), “Democracy and Violence in Brazil”. Comparative Studies in Society and History, 41(4): 691-729.

Самасно, Alberto. (1994a), "Empresarios ilegales y región la gestación de clases dominantes”. Reseña en Delito y Sociedad: Revista de Ciencias Sociales, 4 (5): 25-27.

Самасно, Álvaro. (1994b), “La reforma de la policía Colombiana. Esperanzas frustraciones?”. Nueva Sociedad, 129: 27-40.

Camacho, Álvaro \& Guzmán, Alvaro. (1990), Colombia: Ciudad y violencia. Colombia, Foro Nacional.

Campos, Edmundo. (1985), "Instituição militar no Brasil: Um ensaio bibliográfico". Revista $B I B, 19: 5-19$.

CAmpos, Edmundo. (1978), "A criminalização da marginalidade e a marginalização da criminalidade”. Revista de Administração Pública, 12 (2): 139-161. Disponível em http:// bibliotecadigital.fgv.br/ojs/index.php/rap/article/view/7458/5927.

Campos, Marcelo y Álvarez, Marcos. (2017), "Políticas públicas de segurança, violência e punição no Brasil (2000-2016)”. In: Miceli, Sergio \& MARTINs, Carlos. (orgs.), Sociologia brasileira hoje. São Paulo, Ateliê, pp. 143-217.

Cano, Ignacio. (1997), The use of lethal force by police in Rio de Janeiro. Rio de Janeiro, Iser.

CAno, Ignacio \& DUARTE, Thais. (coords.). (2012), "No sapatinho": a evolução das milícias no Rio de Janeiro (2008-2011). Rio de Janeiro, Fundação Heinrich Böll.

Cano, Ignacio \& Fragoso, José. (1997), "Letalidade da açao policial no Rio de Janeiro: A atuaçao da Justiça Militar”. Revista Brasileira de Ciencias Criminais, 8 (30): 207-233.

CANo, Ignacio \& SANTos, Nilton. (2001), Violência letal, renda e desigualdade social no Brasil. Rio de Janeiro, 7Letras.

CARrión, Fernando. (ed.). (2017), La politica en la violenciay lo politico de la seguridad. Ecuador, Flacso, DRC-CRD.

Carrión, Fernando y Gottsbacher, Markus. (2020), “Border violence in Latin America: an expression of complementary asymmetries". In: Rivera, Liliana \& BADA, Xóchitl (eds.). The Oxford handbook of the sociology of Latin America. Oxford, Oxford University Press [In press]. 
Castillo, Héctor. (2002), “De las bandas a las tribus urbanas: De la transgresión a la nueva identidad social”. Desacatos, 9: 57-71. Disponível em http://www.scielo.org.mx/scielo. php?script=sci_arttext\&pid=S1607-050X2002000100003\&lng=es\&nrm=iso.

Cataño, Gonzalo \& Restrepo, Gabriel. (1989), “The development of sociology in Colombia”. In: Genov, Nikolai. (eds.), National Traditions in Sociology. Londres, Sage.

Снават, Jorge. (1997), La nueva agenda internacional y la politica exterior mexicana. La Politica Exterior de México: enfoques para su análisis. México, El Colegio de México - Instituto Matías Romero de Estudios Diplomáticos.

Chevigny, Paul et al. (1987), Police abuse in Brazil: Summary executions and torture in São Paulo and Rio de Janeiro. Nova York, Americas Watch Committee.

Concha-Eastman, Alberto. (2015), "La sobremortalidad por homicidios en la población de 10 a 29 años. El caso de Colombia”. In: Alvarado, Arturo et al. (ed.), Vidas truncadas: el exceso de homicidios en la juventud de América Latina, 1990-2010. México, El Colegio de México.

Concha-Eastman, Alberto et al. (2020), "Homicides in Latin America and the Caribbean". In: Rivera, Liliana y BADA, Xóchitl. (eds.), The Oxford handbook of the sociology of Latin America. Oxford, Oxford University Press [In press].

Concha-Eastman, Alberto et al. (eds.). (1994). Ciudad y violencias en América Latina, vol. 2. Programa de Gestión Urbana, Oficina Regional para América Latina y el Caribe.

Correa-Cabrera, Guadalupe. (2017), Los Zetas Inc. Criminal corporations, energy and Civil War in Mexico. Austin, University of Texas Press.

Costa, Arthur \& STEPAN, Alfred. (2001), Democratization and the police; crafting in five democratic arenas: "civil society", "political society", "rule of law", "useable state", and "economic society". Conference on Democratic Transition and Consolidation.

Crenzel, Emilio. (2008), La historia politica del Nunca Más: La memoria de las desapariciones en la Argentina. Buenos Aires, Siglo Veintiuno.

CRUz, José et al. (2017), La nueva cara de las pandillas callejeras: El fenómeno de las pandillas en El Salvador. USA, Centro Kimberly Green para América Latina y el Caribe/Instituto Jack D. Gordon.

Cubides, Fernando et al. (1998), La violencia y el municipio colombiano 1980-1997. Colombia, Universidad Nacional de Colombia, CES.

Da matta, Roberto. (1993), “Os discursos da violência no Brasil”. In: Da Mata, Roberto. Conta de mentiroso: Sete ensaios de antropologia brasileira. Rio de Janeiro, Rocco, pp. 175-197.

Datafolha. (1995). A imagem da policía [survey] DAT/sP-RJ95.DEZ-00532, Banco de Dados do Centro de Estudos de Opinião Pública - Cesop-Unicamp. Disponível em http://www. cesop.unicamp.br/site/htm/busca/php, consultado em 27/02/2014.

Davis, Diane. (2020), “Urban violence and the spatial question: The built environmental. Correlates of (in) security in Latin American Cities". In: Rivera, Liliana \& BADA, Xóchitl. 
(eds.), The Oxford handbook of the sociology of Latin America. Oxford, Oxford University Press [In press].

Davis, Diane \& Alvarado, Arturo. (1999), “Descent into chaos. Liberalization, public insecurity and deteriorating rule of law". Working Paper in Local Governance and Democracy, 1: 95-107.

De Freitas, Renan \& Ribeiro, Ludmila. (2013), “Avanços e perspectivas da sociologia no Brasil: uma abordagem comparativa”. Revista Brasileira de Sociologia, 1 (2): 69-113.

Del Olmo, Rosa. (1992), Prohibir o domesticar:politicas de drogas en América Latina. Caracas, Nueva Sociedad.

Del Olmo, Rosa. (1990), “Violencia policial en las calles de Caracas”. In: Del Olmo, Rosa. (ed.). Segunda ruptura criminológica. Caracas, Universidad Central de Venezuela, pp. 217-239.

Del Olmo, Rosa. (1980), Los chigüines de Somoza: un ejemplo de criminalidad latinoamericana. Caracas, Ateneo de Caracas.

Dias, Camila. (2013), PCC: Hegemonia nas prisões e monopólio da violência. São Paulo, Saraiva.

DiAs, Camila et al. (2012), "Políticas penitenciárias e as facções criminosas: uma análise do regime disciplinar diferenciado (RDD) e outras medidas administrativas de controle da População carcerária”. Estudos de Sociologia, 17 (33): 333-351. Disponível em https:// periodicos.fclar.unesp.br/estudos/article/view/5419.

Dias, Camila \& Paes, Bruno. (2018), A guerra: a ascensão do PCC e o mundo do crime no Brasil. Brasil, Edição padrão.

Durán, Angélica. (2020), "Illicit drugs and organized crime in Latin America: New scholarship and the future of alternative policies". In: Rivera, Liliana \& BADA, Xóchitl. (eds.). The Oxford handbook of the sociology of Latin America. Oxford, Oxford University Press [In press].

Dillon, Gláucio. (2011), “A criminologia e as desventuras do jovem dado". O Panorama dos Homicídios no Brasil, 6: 11-30.

Dillon, Gláucio. (1993), "A violência na América Latina”. Revista Brasileira de Ciências Sociais, 8 (21): 22-39.

D’Araujo, Celina et al. (1994), Os Anos de Chumbo. A memória sobre a repressão. Rio de Janeiro, Relume Dumará.

ElWert, Georg et al. (1999), Dynamics of violence: Processes of escalation and De-escalation in violent group conflict. Berlin, Dunker and Humboldt.

Espitia, Victoria et al. (1994). "Vigilancia epidemiológica de los homicidios ocurridos en Cali. Enero a diciembre de 1993. La epidemiología aplicada a la administración pública”. In: Concha-Eastman, Alberto et al. Ciudad y violencias en América Latina. Quito, Programa Gestión Urbana y Alcaldía de Cali, pp. 155-166.

Fals Borda, Orlando. (2001), “Cuarenta años de la sociología en Colombia: problemas y proyecciones”. Revista Colombiana de Sociología, 6 (1): 7-15.

Fausto, Boris. (1984), Crime e cotidiano: a criminalidade em São Paulo, 1880-1924. São Paulo, Edusp. 
Fellini Fascinetto, Rochelle et al. (2020), "As linhagens de descendência acadêmica dos pesquisadores 'pioneiros' nos estudos sobre violência, crime e justiça criminal no Brasil (1970-2018)". Revista Brasileira de Informaçao Bibliográfica e Ciências Sociais, 91:1-39.

Feltrán, Gabriel. (2018), Irmãos: uma história do PCC. São Paulo, Companhia das Letras.

Figueroa, Carlos. (1991), "Guatemala: the recourse of fear”. In: Huggins, Martha (ed.). Vigilantism and the State in Modern Latina America, essays on extralegal violence. Nova York, Praeger, pp. 73-83.

Fontecilla, Alejandro \& SuÁrez, María Eugenia (2014), "Community policing in Mexico: The framework of resistance and conditions of possibility”. Stability: International Journal of Security \& Development, 3 (1): 1-17.

Flores, Carlos. (2013), Historias de polvo y sangre: Génesis y evolución del tráfico de drogas en el estado de Tamaulipas. México, Centro de Investigaciones y Estudios Superiores en Antropología Social.

Franco, Saúl. (1999), El Quinto: No matar. Contextos explicativos sobre la violencia. Santafé de Bogotá, Tercer Mundo, Iepri Universidad Nacional.

Franco, Saúl. (1997). "Violencia y salud en Colombia”. Revista Panamericana de Salud Pública, 1 (2): 93-103.

Frederic, Sabina. (2008), Los usos de la fuerza pública: Debates sobre militares y policías en las ciencias sociales de la democracia. Buenos Aires, Biblioteca Nacional.

FrüHLING, Hugo et al. (2005), Crimen y violencia en América Latina: seguridad ciudadana, democracia y Estado. Bogotá, Fondo de Cultura Económica.

FrüHLING, Hugo et al. (eds.) (2004), Calles más seguras: Estudios de policía comunitaria en América Latina. Nova York, Inter-American Development Bank.

Fuentes, Antonio. (2015), "Narcotráfico y autodefensa comunitaria en 'Tierra Caliente', Michoacán, México”. Ciencia UAT, 10 (1): 68-82.

Hershberg, Eric \& Agüero, Felipe. (2005), Memorias de la represión en el cono sur: visiones en disputa en dictadura y democracia. Argentina, Siglo XxI.

GABALDón, Luis. (2010), “La criminología latinoamericana: temas, perspectivas y políticas públicas en el tránsito del milenio". Espacio Abierto Cuaderno Venezolano de Sociología, 19 (2): 253-272.

Gabaldón, Luis \& Antillano, Andrés. (2007), La policía Venezolana: Desarrollo institucional y perspectivas de reforma al inicio del tercer milenio. Caracas, Comisión Nacional para la Reforma Policial.

Gentilli, Rafael. (1995), Me a va a tener que acompañar. Una visión critica sobre los edictos policiales. Buenos Aires, El Naranjo.

Goldstein, Daniel. (2003), The spectacular city: Violence and performance and in urban Bolivia. NC, Duke University Press Books.

Gómez, Juan et al. (2003), La policía en los Estados de Derecho latinoamericanos. Bogotá, Ediciones Jurídicas Gustavo Ibáñez. 
González, Gustavo. (2004), Jóvenes y delito en la Ciudad de Buenos Aires. Buenos Aires, Unidos por la Justicia.

Guzmán, Germán et al. (1962), La violencia en Colombia: estudio de un proceso social. Colombia, Tercer Mundo.

Hathazy, Paul \& Frederic, Sabina. (2018), “Trabajo policial y política en Argentina: perspectivas y contribuciones de las ciencias sociales". Trabajo y Sociedad, 31: 5-13.

Hinton, Mercedes. (2006). The state on the streets: Police and politics in Argentina and Brazil. Boulder/London, Lynne Rienner Publishers.

Huggins, Martha \& Mesquita, Myriam. (1995), "Scapegoating outsiders: the murder of street youth in modern Brazil”. Policing and Society, 5: 265-280.

Iмвusch, Peter et al. (2011), "Violence research in Latin America and the Caribbean: A literature review". International Journal of Conflict and Violence, 5 (1): 87-154.

JiménEZ, William \& Turizo, Juan. (2011), "Militarización de la policía y policización de las Fuerzas Militares. Revisión del fenómeno a nivel internacional y nacional”. Logos Ciencia \& Tecnologia, 3 (1): 112-126.

JimÉNEZ, William et al. (2000), "Violência, criminalidade, segurança pública e justiça criminal no Brasil: uma bibliografía”. Revista Brasileira de Informação Bibliográfica em Ciências Sociais, 50 (2): 45-123.

Johns, Christina. (1995), “The origins of violence in Mexican society”. In: JonEs, Gareth \& Rodgers, Dennis (ed.). (2009), Youth violence in Latin America. United States of America, Palgrave Macmillan.

Kant, Roberto. (1999), "Polícia, justiça e sociedade no Brasil: Uma abordagem comparativa dos modelos de administração de conflitos no espaço público". Revista de Sociologia e Politica, 13: 23-38.

Kant, Roberto et al. (2000). "Violencia, criminalidade, segurança publica e justicia criminal do Brasil: Uma bibliografía”. Revista Brasileira de Informação Bibliográfica em Ciências Sociais-BIB, 50: 45-123.

Kessler, Gabriel. (2019), "Algunas reflexiones sobre la agenda de investigación de desigualdades en Latinoamérica”. Desacatos, 59: 86-95. Disponível em http://www.scielo.org.mx/ pdf/desacatos/n59/2448-5144-desacatos-59-86.pdf.

KesSLER, Gabriel. (2009), El sentimiento de inseguridad: Sociología de temor al delito. Buenos Aires, Siglo XxI.

Kessler, Gabriel \& OtAmendi, Alejandra. (2020), "Sociology of fear of crime in Latin America”. In: Rivera, Liliana \& BADA, Xóchitl. (eds.), The Oxford handbook of the sociology of Latin America. Oxford, Oxford University Press [In press].

Lemgruber, Julita. (1983), Cemitério dos vivos: análise sociológica de uma prisão de mulheres. Rio de Janeiro, Forense.

Lima, Renato \& Ratton, José (orgs.). (2011), As Ciências Sociais e os pioneiros nos estudos 
sobre crime, violência e direitos humanos no Brasil. São Paulo, Fórum Brasileiro de Segurança Pública/Urbania/Anpocs.

Londoño, Juan y Guerrero, Rodrigo. (2000), “Violencia en América Latina: epidemiología y costos. Asalto al desarrollo”. In: Londoño, Juan et al. (eds.). Violencia en América Latina. Washington, DC, pp. 11-57.

Macaulay, Fiona. (2007), "Knowledge production, framing and criminal justice reform in Latin”. Journal of Latin American Studies, 39 (3): 627-651

Machado, Luiz. (2004), "Sociabilidade violenta: por uma interpretação da criminalidade contemporânea no Brasil urbano”. Sociedade e Estado, 19 (1): 53-84. Disponível em http:// www.scielo.br/scielo.php?script=sci_arttext $\&$ pid $=$ S0102-69922004000100004\&lng=e $\mathrm{n} \& \mathrm{nrm}=\mathrm{iso}$.

Maldonado, Salvador. (2010), Los márgenes del estado mexicano: Territorios ilegales, desarrollo y violencia en Michoacán. Michoacán, El Colegio de Michoacán.

Manzano, Liliana. (2020), "Violent victimization in poor neighborhoods of Bogotá, Lima, and Santiago: Empirical test of the Social Disorganization and the Collective Efficacy Theories". In: RiverA, Liliana \& BADA, Xóchitl (eds.). The Oxford handbook of the sociology of Latin America. Oxford, Oxford University Press [In press].

MARín, Juan. (1996), Los hechos armados. Argentina 1973-1976: la acumulación primitiva del genocidio. Buenos Aires, Programa Investigación Cambio Social.

Martínez, Beatriz. (1999), La policía en México: ¿orden social o criminalidad?. DF, Planeta.

Masur, Jandira. (1994), “Drogas ¿Cuál es el problema?”. Delito y Sociedad: Revista de Ciencias Sociales, 4: 13-15.

Méndez, Luz. (2013), La erradicación de la violencia contra las mujeres y el papel de la Policía Nacional Civil. Guatemala, FyGeditores.

MÉndez, Luz y BARrios, Walda. (2010), “Caminos recorridos: luchas y situación de las mujeres a trece años de los Acuerdos de paz”. In: Monzón, Ana (coord.). Antología del pensamiento critico guatemalteco contemporáneo. Buenos Aires, Clacso, pp. 751-760.

Mesquita, Paulo. (2004), "Policiamento comunitário e prevenção do crime: a visão dos coronéis da Polícia Militar". São Paulo em Perspectiva, 18 (1): 103-110.

Mesquita Neto, Paulo de. (2004), "Policiamento comunitário e prevenção do crime: a visão dos coronéis da Polícia Militar”. São Paulo em Perspectiva, 18 (1): 103-110.

Mingardi, Guaracy. (1992), Tiras, gansos e trutas: Cotidiano e reforma na polícia civil. São Paulo, Aberta.

Misse, Michel. (2019), "Alguns aspectos analíticos nas pesquisas da violência na América Latina”. Estudos Avançados, 33 (96): 23-38. Diponível em http://www.scielo.br/scielo. php?script=sci_arttext\&pid=S0103-40142019000200023\&lng=en\&nrm=iso.

Misse, Michel. (1997), “As ligações perigosas: Mercado informal ilegal, narcotráfico e violência no Rio de Janeiro". Contemporaneidade e Educação, 2 (1): 93-116. 
Misse, Michel (1973), Delinquência juvenil na Guanabara: uma introdução sociológica. In: Cavallieri, Paulo Fernando et al. Tribunal de Justiça do Estado de Guanabara. Rio de Janeiro, Juizado de Menores do Rio de Janeiro.

Misse, Michel \& CARrión, Fernando. (2011), "Crime organizado e crime comum no Rio de Janeiro: Diferenças e afinidades”. Revista de Sociologia e Política, 19 (40): 13-25.

Misse, Michel \& Motta, Dilson. (1979), Crime: o social pela culatra. Rio de Janeiro, Achiamé. Monarrez, Julia y Tabuenca, María. (2007), Bordeando la violencia contra las mujeres en la frontera norte de Mexico. Ciudad Juárez, Colef-Porrúa.

Muniz, Jacqueline et al. (1997), "Resistências e dificuldades de um programa de policiamento comunitário". Tempo Social: Revista de Sociologia, 9 (1): 197-213. Disponível em http:// www.revistas.usp.br/ts/article/view/86552/89391.

Mura, Gustavo. (1997). Pecado capital: cómo opera la mafia del tráfico de niños en La Argentina. Buenos Aires, Sudamericana.

NACIf, Mina Jorge. (1986), La policía en la historia de la ciudad de México. México, Departamento del Distrito Federal.

Nelligan, Mauricio. (1988), Mujeres que matan: prostitución y homicidio femenil en México. México, Edamex.

Ortiz, Carlos. (1998), “El homicidio en Colombia 1959-/1997”. In: Cubides, Fernando. La violencia en el municipio colombiano 1980-1997. Colombia, Universidad Nacional de Colombia, CEs.

PaIXão, Antônio Luiz. (1982a), "Police organization in a metropolitan area”. Dados: Revista Brasileira de Ciências Sociais, 25 (2): 63-85.

PAIX̃̃o, Antônio Luiz. (1982b), "A Organização Policial numa área Metropolitana”. Dados: Revista Brasileira de Ciências Sociais, 25 (1): 63-85.

PÉcaut, Daniel. (1987), Orden y violencia en Colombia. Medellín, Siglo XXI.

Pegoraro, Juan. (1997), "Las relaciones sociedad-Estado y el paradigma de la inseguridad". Delito y Sociedad: Revista de Ciencias Sociales, 9 (10): 9-55.

Pegoraro, Juan. (1992), “Delito y sociedad”. Revista de Ciencias Sociales, 1: 1-2.

Pinheiro, Paulo. (1984), Escritos indignados: polícia, prisóes e política no Estado autoritário: no 20a aniversário do Regime de Exceção (1964-1984). São Paulo, Brasiliense.

Pinheiro, Paulo (org.). (1983), Crime, violência e poder. Brasiliense.

Pinheiro, Paulo et al. (1991). "Violência fatal: Conflitos policiais em São Paulo (81-89)". Revista USP, 9: 95-112.

Piquet, Carneiro. (2000), Violent crime in Latin America cities: Rio de Janeiro/São Paulo. Brasil, mimeo.

Quiroz, Alfonso y Quiroz, Raúl. (1970), El costo social del delito en México: symposium sobre el costo del crimen y la defensa social contra el mismo. México, Librería y Ediciones Botas.

Ratton, José. (2018), “Crime, polícia e sistema de justiça no Brasil contemporâneo: uma car- 
tografia (incompleta) dos consensos e dissensos da produção recente das Ciências Sociais". Revista Brasileira de Informação Bibliográfica em Ciências Sociais, 84: 5-12.

Reguillo, Rossana. (2000), “Los laberintos del miedo. Un recorrido para fin de siglo”. Revista de Estudios Sociales, 5: 63-72.

Restrepo, Riaza et al. (1994). "La imagen social de la policía en Medellín”. Estudios Politicos, 5: 47-67.

Rico, José \& Chinchilla, Laura. (2002), Seguridad ciudadana en América Latina. Ciudad de México, México Siglo Xxi.

Rifiotis, Theophilos. (1999), “Violência policial e imprensa: o caso da Favela Naval." São Paulo em Perspectiva, 13(4): 28-41.

Rodgers, Dennis. (2017), "Bróderes in arms: Gangs and the socialization of violence in Nicaragua". Journal of Peace Research, 54 (5): 648-660.

Romero, Mauricio et al. (2007), Parapolítica. La ruta de la expansión paramilitar y los acuerdos politicos. Colombia, Cedec-Corporacion Arcoiris.

Rotker, Susana (ed.), (2000), Ciudadanías del miedo. Caracas, Nueva Sociedad.

Ruíz, Rafael. (1998), Criminalidad y mal gobierno. México, Sansores y Allure.

SAÍn, Marcelo. (2002), Seguridad, democracia y reforma del sistema policial en la Argentina. Buenos Aires, Fondo de Cultura Económica.

Salla, Fernando. (2001), "Brazil's Prison Debacle". Nacla Reporting on the Americas, 35 (2): 8.

SAlla, Fernando. (2006), "As rebeliões nas prisões: novos significados a partir da experiência brasileira”. Sociologias, 16: 274-307. Disponível em http://www.scielo.br/scielo. php?script=sci_arttext\&pid=S1517-45222006000200011\&lng=en\&nrm=iso.

SÁnchez, Gónzalo. (2013), ¡Basta ya! Colombia: memorias de guerra y dignidad. Colombia, Informe general Grupo de Memoria Histórica, CNMH-DPS-Prosperidad para todos.

SÁnchez, Gónzalo (coord.). (1987), Colombia: violencia y democracia. Colombia, Comisión de estudios sobre la violencia-Iepri-Universida Nacional de Colombia.

SANDOVAL, Mary. (2014), "Investigación sociológica y conflicto armado en Colombia”. Revista Colombiana de Sociología, 37 (1): 99-120.

Santacruz, María et al. (2001), Barrio Adentro. La Solidaridad Violenta de las Pandillas. San Salvador, Uidop.

SANTOS, José y BARreira, Cesar. (2016), “Introdução: a construção de um campo intelectual: violência e segurança cidadã na América Latina”. In: SANTos, José \& BARreira, Cesar (orgs.). Paradoxos da segurança cidadã. Porto Alegre, Tomo Editorial, pp. 9-40.

Schmid, Robert. (1996) “La corrupción en la policía preventiva del Distrito Federal en México”. In: Waldmann, Peter. (ed.). Justicia en la calle. Ensayos sobre la policía en América Latina. Buenos Aires, Biblioteca jurídica Diké.

SoAres, Luis. (2000), Meu casaco de general: 500 dias no front da segurança pública do Rio de Janeiro. São Paulo, Companhia das Letras. 
SUÁREZ, Andrés. (1999), "Configuraciones y dinámicas de la violencia organizada en Colombia 1987-1997”. Revista Colombiana de Sociología, 4 (1): 15-33.

TisCORNIA, Sofía. (1997), "Violencia policial”. In: Informe anual sobre la situación de los derechos bumanos en la Argentina. Argentina, Centro de Estudios Legales y Sociales. Disponível em https://www.cels.org.ar/web/capitulos/violencia-policial/.

Tulchin, Joseph et al. (eds.), (2003), Crime and violence in Latin America: Citizen security, democracy and the State. us, Woodrow Wilson Center Press.

Undp. (2010), Human Development Report 2010. United Nations Development Programme Commentary.

Villamizar, Darío. (2017), Las guerrillas en Colombia: una historia desde los orígenes hasta los confines. Colombia, Penguin Random House.

Vizcaíno, Milcíades et al. (2002), "Homicidios: una mirada desde los actores". Revista Colombiana de Sociología, 7 (1): 145-171.

Waiselfisz, Julio. (1998), Mapa da violência: os jovens do Brasil. Rio de Janeiro, Garamond, Instituto Ayrton Senna y Unesco.

Willis, Graham. (2013), The killing consensus: Homicide detectives, police that kill and organized crime in São Paulo, Brazil. Massachusetts, Doctoral Dissertation. Massachusetts Institute of Technology.

Word Health Organization. (Who), (2014), Developing countries in Latin America and the Caribbean have world's highest homicide rates. Pan American Health Organization

Zaluar, Alba. (2000), "Perverse integration: Drug trafficking and youth in the favelas of Rio de Janeiro". Journal of International Affairs, 53 (2): 654-71.

Zaluar, Alba. (1999), "Violência e crime”. In: Miceli, Sergio (ed.). O que ler nas ciências sociais brasileiras. São Paulo, Sumaré/Anpocs, pp. 13-107.

ZALUAR, Alba. (1985), A máquina e a revolta: as organizações populares e o significado da pobreza. São Paulo, Brasiliense.

Zavaleta, Alfredo et al. (2016), "Una aproximación a las relaciones entre policías y jóvenes en América Latina”. Politica y Gobierno, 23 (1): 201-229.

Zinni, Héctor. (1992), La mafia en Argentina. Rosario, Sta. Fé, Centro Editorial. 


\section{Resumen}

La Sociología del crimen y la violencia en América Latina: un campo fragmentado

El propósito del presente artículo es establecer una genealogía de los estudios sobre Sociología del crimen y la violencia en América Latina, particularmente desde los años sesenta del siglo Xx, cuando emerge un cuerpo de la literatura que tiene las características de una subdisciplina, con una comunidad de expertos que genera conocimientos y promueve un campo analítico propio, autónomo. En el presente estudio proporcionamos una muestra representativa de los estudios en el subcontinente y examinamos sus bases teóricas y sus métodos. La formación de estas comunidades ha sido impulsada por el inexorable desenvolvimiento del crimen. Con la excepción de Colombia desde la mitad del siglo pasado, el resto de los países pasaron de bajos niveles de criminalidad al incremento exponencial de múltiples formas de violencia y a la multiplicación de actores ilícitos. Los desafíos han crecido con mayor velocidad con las explicaciones nacionales fragmentadas. Palabras-clave: Sociología del crimen y la violencia en América Latina; Historia de la Sociología; delitos.

\section{Abstract}

The Sociology of crime and violence in Latin America: a fragmented field

The purpose of this article is to establish a genealogy of studies on the Sociology of crime and violence in Latin America, particularly since the 1960s of the 20th century, when a body of literature emerges with clear characteristics of a sub-discipline, with a community of knowledge that promotes an autonomous analytical field. The generating process of the creation of these communities has been the inexorable development of crime. With the exception of Colombia since the mid-Xx century, the rest of the countries went from low levels of crime to the exponential increase in multiple forms of violence and the multiplication of illegal actors. The challenges have grown faster with fragmented national explanations. In the present study we provide a representative sample of the studies in the subcontinent and examine their theoretical bases and methods. Keywords: Sociology of crime and violence in Latin America; History of Sociology; Crimes.

\section{Resumo}

A sociologia do crime e da violência na América Latina: um campo fragmentado

O objetivo deste artigo é estabelecer uma genealogia dos estudos sobre a sociologia do crime e da violência na América Latina, particularmente desde os anos 60 do século XX, quando surge um corpo de literatura com características claras de uma subdisciplina, com uma comunidade de conhecimento que promove seu próprio campo analítico autônomo. O processo gerador da criação dessas comunidades tem sido o desenvolvimento inexorável do crime. Com exceção da Colômbia, o resto dos países passou de baixos níveis de criminalidade para o aumento exponencial de múltiplas formas de violência e a multiplicação de atores ilegais. Os desafios cresceram mais rapidamente com explicações nacionais fragmentadas. No presente estudo, fornecemos uma 
amostra representativa dos estudos no subcontinente e examinamos criticamente suas bases teórico-metodológicas.

Palavras-chave: Sociologia do crime e violência na América Latina; História da sociologia; Crimes.

Texto recebido em 22/9/2020 e aprovado em 26/9/2020.

DOI: 10.11606/0103-2070.ts.2020.175010.

Arturo Alvarado M. é sociólogo, professor-pesquisador do El Colegio de México. Especialista em temas de segurança pública, polícia, governança democrática e crime. Foi pesquisador visitante na Universidade de São Paulo (USP/NEvE), Brasil (2019), assim como professor visitante na Universidade de Princeton, EUA. Seus artigos mais recentes são: “Organizaciones criminales en América Latina: una discusión conceptual y un marco comparativo para su reinterpretación", publicado na Revista Brasileira de Sociologia-RBS, 2019 (vol. 7, n. 17, pp. 11-32), e "Reformas policiales y la construcción de un estado de derecho. El caso de la policía de vecinal de Ciudad Nezahualcóyotl”, Desacatos. Revista de Ciencias Sociales, 2019 (vol. 19, n. 60, pp. 34-57). E-mail: alvarado@colmex.mx. 


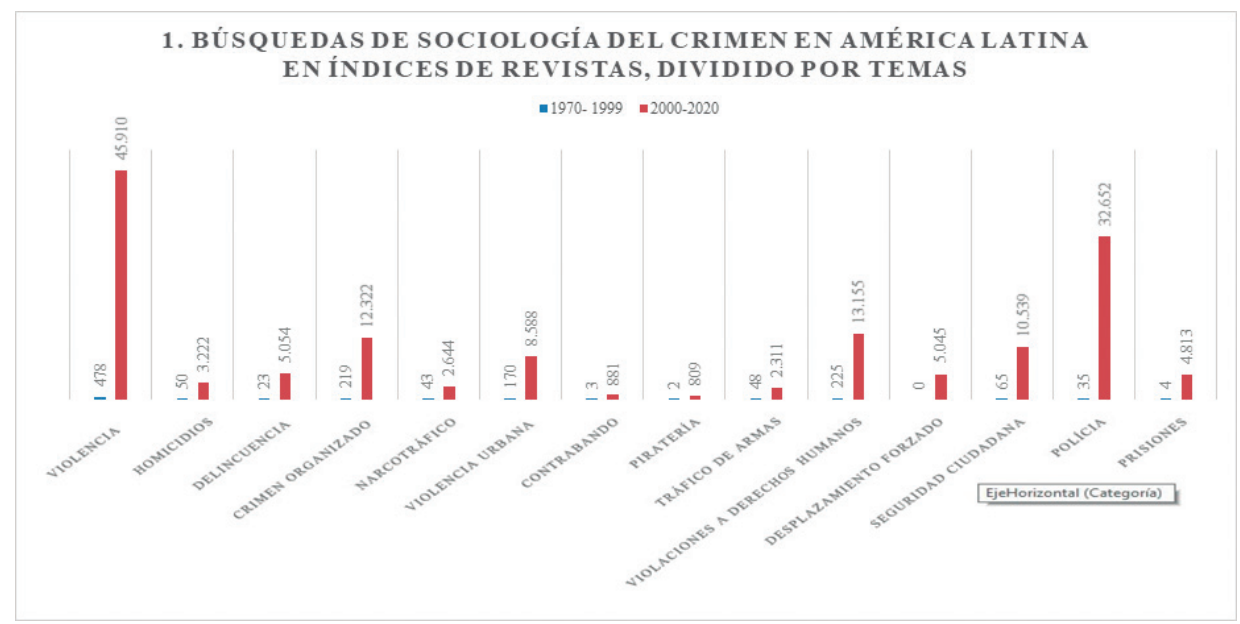

Fuente: Elaboración propia con base en búsquedas bibliográficas.

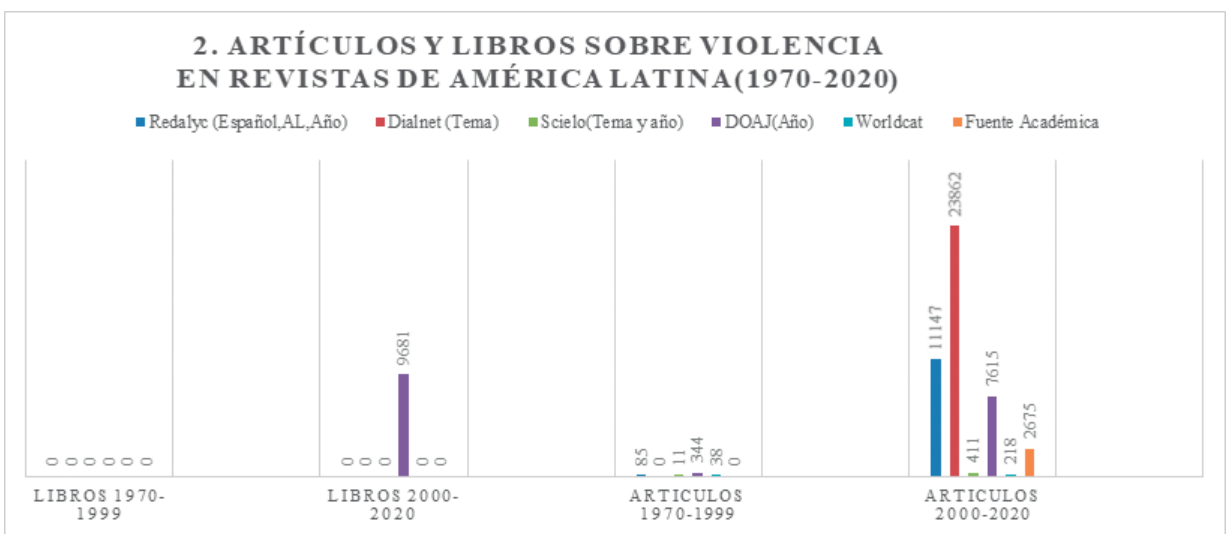

Fuente: Elaboración propia con base en búsquedas bibliográficas. 
ANEXO

Búsquedas sobre sociologia del crimen en revistas de ciencias socialies en América Latina (1 de 2)

\begin{tabular}{|c|c|c|c|c|c|}
\hline \multirow{2}{*}{ TEMA } & \multirow{2}{*}{ Fuente } & LiBros & \multicolumn{3}{|c|}{ ARTíCULOS } \\
\hline & & 1970-1999 & $2000-2020$ & $1970-1999$ & $2000-2020$ \\
\hline \multirow{6}{*}{ Violencia } & Redalyc (Español, AL, Año) & S/D & S/D & 85 & 11,147 \\
\hline & Dialnet (Tema) & $S / D$ & $S / D$ & $S / D$ & 23862 \\
\hline & Scielo (Tema y año) & $S / D$ & $S / D$ & 11 & 411 \\
\hline & DOAJ (Año) & $S / D$ & 9681 & 344 & 7615 \\
\hline & worldcat & $S / D$ & $S / D$ & 38 & 218 \\
\hline & Fuente Académica & $S / D$ & $\mathrm{~S} / \mathrm{D}$ & $\mathrm{S} / \mathrm{D}$ & 2657 \\
\hline \multirow{6}{*}{ Homicidios } & Redalyc (Español, AL, Año) & S/D & S/D & S/D & 1487 \\
\hline & Dialnet (Tema) & $S / D$ & 37 & $S / D$ & 882 \\
\hline & Scielo (Tema y año) & $S / D$ & $\mathrm{~S} / \mathrm{D}$ & 50 & 495 \\
\hline & DOAJ (Año) & S/D & S/D & S/D & 224 \\
\hline & worldcat & $\mathrm{S} / \mathrm{D}$ & S/D & $\mathrm{S} / \mathrm{D}$ & 4 \\
\hline & Fuente Académica & S/D & S/D & $\mathrm{S} / \mathrm{D}$ & 130 \\
\hline \multirow{6}{*}{ Delincuencia } & Redalyc & $S / D$ & $S / D$ & 10 & 860 \\
\hline & Dialnet & S/D & 384 & $S / D$ & 3087 \\
\hline & Scielo & $S / D$ & $S / D$ & 4 & 357 \\
\hline & Doaj & S/D & $\mathrm{S} / \mathrm{D}$ & $\mathrm{S} / \mathrm{D}$ & 515 \\
\hline & Worldcat & 9 & 58 & 1 & 15 \\
\hline & Fuente Académica & $\mathrm{S} / \mathrm{D}$ & S/D & 8 & 220 \\
\hline \multirow{6}{*}{$\begin{array}{l}\text { Crimen } \\
\text { Organizado }\end{array}$} & Redalyc & $S / D$ & S/D & 217 & 10726 \\
\hline & Dialnet & S/D & S/D & S/D & 1111 \\
\hline & Scielo & $S / D$ & S/D & 1 & 173 \\
\hline & Doaj & $S / D$ & S/D & $\mathrm{S} / \mathrm{D}$ & 189 \\
\hline & Worldcat & 5 & 17 & 1 & 9 \\
\hline & Fuente Académica & $\mathrm{S} / \mathrm{D}$ & S/D & S/D & 114 \\
\hline \multirow{6}{*}{ Narcotráfico } & Redalyc & $\mathrm{S} / \mathrm{D}$ & S/D & 18 & 560 \\
\hline & Dialnet & $S / D$ & 34 & $\mathrm{~S} / \mathrm{D}$ & 1123 \\
\hline & Scielo & S/D & S/D & 10 & 316 \\
\hline & Doaj & $S / D$ & S/D & $\mathrm{S} / \mathrm{D}$ & 419 \\
\hline & Worldcat & 32 & 34 & 12 & 21 \\
\hline & Fuente Académica & $\mathrm{S} / \mathrm{D}$ & S/D & 3 & 205 \\
\hline \multirow{6}{*}{$\begin{array}{l}\text { Violencia } \\
\text { Urbana }\end{array}$} & Redalyc & $\mathrm{S} / \mathrm{D}$ & S/D & 149 & 7238 \\
\hline & Dialnet & $\mathrm{S} / \mathrm{D}$ & 18 & $\mathrm{~S} / \mathrm{D}$ & 783 \\
\hline & Scielo & S/D & S/D & 20 & 315 \\
\hline & Doaj & $\mathrm{S} / \mathrm{D}$ & S/D & $\mathrm{S} / \mathrm{D}$ & 196 \\
\hline & Worldcat & 17 & 61 & $\mathrm{~S} / \mathrm{D}$ & 9 \\
\hline & Fuente Académica & $\mathrm{S} / \mathrm{D}$ & S/D & 1 & 47 \\
\hline \multirow{6}{*}{ Contraband } & Redalyc & $\mathrm{S} / \mathrm{D}$ & $\mathrm{S} / \mathrm{D}$ & 3 & 141 \\
\hline & Dialnet & $\mathrm{S} / \mathrm{D}$ & 55 & S/D & 496 \\
\hline & Scielo & $\mathrm{S} / \mathrm{D}$ & S/D & S/D & 6 \\
\hline & Doaj & $\mathrm{S} / \mathrm{D}$ & S/D & $\mathrm{S} / \mathrm{D}$ & 203 \\
\hline & Worldcat & $S / D$ & 99 & $\mathrm{~S} / \mathrm{D}$ & 5 \\
\hline & Fuente Académica & $S / D$ & $S / D$ & $S / D$ & 30 \\
\hline
\end{tabular}


La Sociología del crimen y la violencia en América Latina, pp. 67-107

ANEXO

Búsquedas sobre sociología del crimen en revistas de ciencias socialies en América Latina (2 de 2)

\begin{tabular}{|c|c|c|c|c|c|}
\hline \multirow{2}{*}{ Tema } & \multirow{2}{*}{ Fuente } & \multirow{2}{*}{$\begin{array}{c}\text { Libros } \\
1970-1999\end{array}$} & \multicolumn{3}{|c|}{ ARtículos } \\
\hline & & & $2000-2020$ & $1970-1999$ & $2000-2020$ \\
\hline \multirow{6}{*}{ Preatria } & Redalyc & $\mathrm{S} / \mathrm{D}$ & $\mathrm{S} / \mathrm{D}$ & 2 & 4 \\
\hline & Dialnet & S/D & 51 & $S / D$ & 739 \\
\hline & Scielo & S/D & S/D & $\mathrm{S} / \mathrm{D}$ & 1 \\
\hline & Doaj & S/D & S/D & $S / D$ & 11 \\
\hline & Worldcat & $\mathrm{S} / \mathrm{D}$ & 114 & $S / D$ & 4 \\
\hline & Fuente Académica & S/D & S/D & $\mathrm{S} / \mathrm{D}$ & 50 \\
\hline \multirow{6}{*}{$\begin{array}{l}\text { Tráfico de } \\
\text { armas }\end{array}$} & Redalyc & S/D & $\mathrm{S} / \mathrm{D}$ & 48 & 2174 \\
\hline & Dialnet & S/D & 6 & $S / D$ & 125 \\
\hline & Scielo & S/D & S/D & $\mathrm{S} / \mathrm{D}$ & 2 \\
\hline & Doaj & $\mathrm{S} / \mathrm{D}$ & $\mathrm{S} / \mathrm{D}$ & $\mathrm{S} / \mathrm{D}$ & 2 \\
\hline & Worldcat & S/D & 59 & S/D & 2 \\
\hline & Fuente Académica & S/D & $\mathrm{S} / \mathrm{D}$ & S/D & 6 \\
\hline \multirow{6}{*}{$\begin{array}{l}\text { Violaciones a } \\
\text { derechos humanos }\end{array}$} & Redalyc & S/D & S/D & 162 & 11886 \\
\hline & Dialnet & S/D & 49 & $\mathrm{~S} / \mathrm{D}$ & 1185 \\
\hline & Scielo & S/D & S/D & $\mathrm{S} / \mathrm{D}$ & 10 \\
\hline & Doaj & S/D & $\mathrm{S} / \mathrm{D}$ & $\mathrm{S} / \mathrm{D}$ & S/D \\
\hline & Worldcat & $\mathrm{S} / \mathrm{D}$ & $S / D$ & 63 & 3 \\
\hline & Fuente Académica & S/D & S/D & $\mathrm{S} / \mathrm{D}$ & 71 \\
\hline \multirow{6}{*}{$\begin{array}{l}\text { Desplazamiento } \\
\text { forzado }\end{array}$} & Redalyc & S/D & $\mathrm{S} / \mathrm{D}$ & S/D & 3925 \\
\hline & Dialnet & S/D & 28 & S/D & 631 \\
\hline & Scielo & S/D & $\mathrm{S} / \mathrm{D}$ & $S / D$ & 4 \\
\hline & Doaj & $\mathrm{S} / \mathrm{D}$ & $\mathrm{S} / \mathrm{D}$ & $S / D$ & 320 \\
\hline & Worldcat & S/D & 402 & S/D & 10 \\
\hline & Fuente Académica & S/D & S/D & $S / D$ & 155 \\
\hline \multirow{6}{*}{$\begin{array}{l}\text { Seguridad } \\
\text { ciudadana }\end{array}$} & Redalyc & S/D & S/D & 65 & 7724 \\
\hline & Dialnet & S/D & 156 & $S / D$ & 1884 \\
\hline & Scielo & S/D & S/D & $\mathrm{S} / \mathrm{D}$ & 7 \\
\hline & Doaj & S/D & S/D & S/D & 278 \\
\hline & Worldcat & S/D & 620 & $\mathrm{~S} / \mathrm{D}$ & 581 \\
\hline & Fuente Académica & S/D & S/D & S/D & 65 \\
\hline \multirow{6}{*}{ Polícia } & Redalyc & S/D & S/D & 25 & 1910 \\
\hline & Dialnet & $\mathrm{S} / \mathrm{D}$ & 512 & S/D & 30266 \\
\hline & Scielo & S/D & S/D & 10 & 105 \\
\hline & Doaj & $\mathrm{S} / \mathrm{D}$ & $\mathrm{S} / \mathrm{D}$ & S/D & 84 \\
\hline & Worldcat & S/D & 1383 & S/D & 23 \\
\hline & Fuente Académica & S/D & S/D & S/D & 264 \\
\hline \multirow{6}{*}{ Prisiones } & Redalyc & S/D & S/D & 4 & 1363 \\
\hline & Dialnet & S/D & 250 & S/D & 2776 \\
\hline & Scielo & S/D & S/D & S/D & 8 \\
\hline & Doaj & S/D & S/D & S/D & 269 \\
\hline & Worldcat & S/D & 559 & S/D & 362 \\
\hline & Fuente Académica & $\mathrm{S} / \mathrm{D}$ & $S / D$ & $\mathrm{~S} / \mathrm{D}$ & 35 \\
\hline
\end{tabular}

Fuente: Elaboración del autor con base en portales de internet señalados en el texto. 
ANEXO

Búsquedas sobre sociologia del crimen en revistas de ciencias socialies en América Latina (por pais)

\begin{tabular}{c|l|c|c|c|c}
\hline \multirow{2}{*}{ Tema } & \multicolumn{1}{|c|}{ Fuente } & Libros & \multicolumn{3}{c}{ Artículos } \\
\cline { 3 - 6 } & & $1970-1999$ & $2000-2020$ & $1970-1999$ & $2000-2020$ \\
\hline \multirow{3}{*}{ México } & Redalyc (Español, AL, Año, país) & S/D & S/D & 51 & 3860 \\
\cline { 2 - 6 } & Scielo & S/D & S/D & S/D & 96 \\
\hline \multirow{2}{*}{ Argentina } & Redalyc (Español, AL, Año, país) & S/D & S/D & 2 & 1013 \\
\cline { 2 - 6 } & Scielo & S/D & S/D & S/D & 60 \\
\hline \multirow{2}{*}{ Brasil } & Redalyc (Español, AL, Año, país) & S/D & S/D & 3 & 663 \\
\cline { 2 - 6 } & Scielo & S/D & S/D & 11 & 100 \\
\hline \multirow{2}{*}{ Colombia } & Redalyc (Español, AL, Año, país) & S/D & S/D & 19 & 3110 \\
\cline { 2 - 6 } & Scielo & S/D & S/D & S/D & 155 \\
\hline
\end{tabular}

Fuente: Elaboración del autor con base en portales de internet señalados en el texto. 